University of Rhode Island

DigitalCommons@URI

Open Access Dissertations

1993

\title{
The Relationship Between Prior Abuse and Personality Functioning in Adolescent Psychiatric Inpatients
}

Kimberly Shaunesey

University of Rhode Island

Follow this and additional works at: https://digitalcommons.uri.edu/oa_diss

\section{Recommended Citation}

Shaunesey, Kimberly, "The Relationship Between Prior Abuse and Personality Functioning in Adolescent Psychiatric Inpatients" (1993). Open Access Dissertations. Paper 1067.

https://digitalcommons.uri.edu/oa_diss/1067

This Dissertation is brought to you for free and open access by DigitalCommons@URI. It has been accepted for inclusion in Open Access Dissertations by an authorized administrator of DigitalCommons@URI. For more information, please contact digitalcommons-group@uri.edu. 


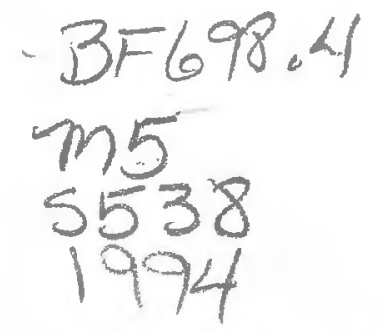

THE RELATIONSHIP BETWEEN PRIOR ABUSE AND PERSONALITY FUNCTIONING IN ADOLESCENT PSYCHIATRIC INPATIENTS BY

KIMBERLY SHAUNESEY

A DISSERTATION SUBMITTED IN PARTIAL FULFILLMENT OF THE REQUIREMENTS FOR THE DEGREE OF DOCTOR OF PHILOSOPHY IN PSYCHOLOGY

1993<smiles>[AsH2]=[AsH2][Te]</smiles> 


\section{Abstract}

Previous research has indicated that a history of abuse is related to later problems in personality functioning in adults. In addition, the literature has suggested that abused adolescents also experience more family dysfunction. In the present investigation, 541 subjects' records were drawn from consecutive admissions to the adolescent inpatient service of a private psychiatric hospital. Psychological records which contained copies of the scored Minnesota Multiphasic Personality Inventory (MMPI) and the Family Assessment Device (FAD) were reviewed. Corresponding medical charts were obtained, from which abuse histories, the Hollingshead Four Factor Index of Social Status, and other demographic information were recorded. A 2 (sex) by 4 (abuse category: none, physical, sexual, both) repeated measures multivariate analysis of variance found that a history of both physical and sexual abuse was related to more personality pathology as measured by the MMPI. There was also some evidence that sexual abuse alone may be related to more difficulties as well. In addition, a repeated measures multivariate analysis of variance revealed that difficulties in personality functioning are also related to more perceived family dysfunction by the adolescent. Although there were no differences in personality functioning for incest and nonfamilial abuse victims, incest victims did report fewer problems in family functioning. Moreover, by conducting 
discriminant function analyses, this study also provided support that the MMPI is an effective tool for identifying abused individuals on the basis of their responses to a number of specific MMPI items. Furthermore, the consequences of using clinically defined cut-off points to define psychopathology and of focusing on an adolescent inpatient population are discussed. Finally, the present study built on the findings of previous investigations as both physical and sexual abuse victims, and males were included in the sample. 


\section{LIST OF TABLES}

1. Description of MMPI standard Scales.............20

2. Number of Males and Females in Each Abuse Group.....25

3. Means for the Significant Simple Effects Tests for Differences among the Four Abuse Groups for the 14 MMPI Scales............................. 8

4. Means for the Significant Simple Effects Tests for Males and Females on the Scaled MMPI Dimensions.....30

5. Means for the Simple Effects Tests and Tukey Follow-up Tests for 3 FAD Groups on 14 Scaled MMPI Dimensions...........................

6. Standardized Canonical Discriminant Function Coefficients for the 27 Selected Items of the MMPI used to Classify Sexually and Nonsexually Abused Females and Group Centroids for the Significant Linear Discriminant Function.....................

7. Standardized Canonical Discriminant Function Coefficients for the 31 selected Items of the MMPI used to Classify Subjects into Abuse Category and Group Centroids for the Two Significant Linear Discriminant Functions..................40

8. Standardized Canonical Discriminant Function Coefficients for the 4 MMPI Standard Scales used to Classify Subjects into Abuse Group and Group Centroids for the Significant Linear Discriminant Function. .........................42

9. Significant MMPI Scales for the Chi Square Analyses for Abuse Category by Pathology, using 70 as the Cut-off Score Defining Pathology..............44

10. Significant MMPI Scales for the Chi Square Analyses for Abuse Category by Pathology, using 65 as the Cut-off Score Defining Pathology..............45 


\section{LIST OF FIGURES}

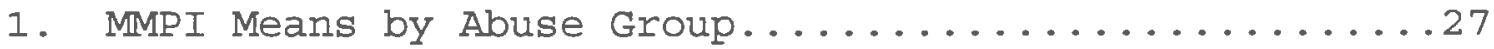

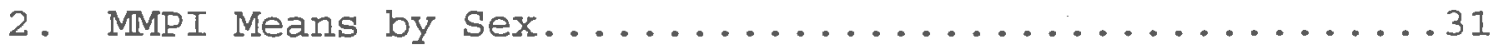

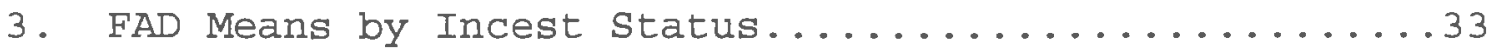

4. MMPI Means by FAD Group................................ 


\section{TABLE OF CONTENTS}

Abstract.............ii

List of Tables...........

List of Figures...........vi

Introduction. ...........

Method...............16

subjects.............16

Measures...............17

Procedure..............23

Results.............24

Discussion............43

Literature Cited........68

Bibliography.......... . . 74 
The Relationship Between Prior Abuse and Personality Functioning in Adolescent Psychiatric Inpatients

Statement of the Problem

The purpose of the present study is to investigate whether individuals who have experienced either physical or sexual abuse as children demonstrate difficulties in personality functioning during adolescence. It is predicted that:

1) physically and sexually abused adolescents will have significantly more pathological (higher) MMPI profiles than nonabused adolescents

2 ) adolescents who have experienced both physical and sexual abuse will have more pathological MMPI profiles than adolescents who have experienced only physical or only sexual abuse

3 ) sexually abused adolescents who were abused by a family member will exhibit more pathological MMPI profiles than adolescents who were abused by a nonfamily member.

Moreover, it is also predicted that:

4) abused adolescents will report more family dysfunction than nonabused adolescents

5) adolescents who rate their families as more dysfunctional will have more pathological MMPI profiles than adolescents who perceive their families as more functional

6) abused adolescents can be identified on the basis of their personality profiles and their perception of family functioning

Justification and Significance

History of Physical Abuse

Child physical abuse has existed since the beginning of time. Stories of ancient Romans exposing unwanted infants to death on hillsides, and of the 1900's sweatshops 
in the United States where children worked long and difficult hours are only two historical examples. One reason why such atrocities were allowed to exist was because children used to be considered the property of their parents to dispose of as they wished (Starr, 1988; Kempe \& Helfer, 1980). It was not until the 1940's when an article by Coffey and Silverman (1945, as cited in Starr, 1988) suggested that many of the injuries pediatricians saw may have actually been inflicted by parents, and Henry Kempe, in 1962, coined the term "the battered child" in order to shock and motivate apathetic professionals, (Starr, 1988) that researchers started to become interested in child abuse and neglect. In fact, it has only been in the past 20 years that investigators have taken an active interest in the area (Starr, 1988; Carmen, Rieker, \& Mills, 1984; Blumberg, 1981).

A Federal Child Abuse Prevention and Treatment Act was passed in 1974 and amended in 1978. This act ensured states assistance in developing abuse and neglect programs, support for research, and established the National Center for Child Abuse and Neglect, and other human service agencies (Starr, 1988; Kadushin \& Martin, 1981). An abundance of Child Abuse and Neglect legislation has been enacted since that time, but has done little to offset the ever increasing rates. It is estimated that 1.7 million children are physically abused each year in this country alone (Kadushin \& Martin, 1981). However, prevalence rates 
are often inaccurate as child abuse often goes unreported and is difficult to prove. Furthermore, $15 \%$ of the children under the age of 5 , seen in emergency rooms, show evidence of abuse (Mayhall \& Norgard, 1983). However, despite its prevalence child abuse has not been adequately defined, legally or morally, preventing reliable studies from being completed.

Definition of Physical Abuse

Cross-cultural contexts must be considered when discussing abuse. What is thought to be abusive in one culture may not be considered so in another. For example, the Vietnamese have a practice called "cao gio", in which they vigorously rub metal coins against their bodies in order to reduce fever, chills, and headaches. The rubbing often leaves bruises which at first were interpreted as signs of abuse by Westerners. New Guineans were appalled when American anthropologists, living among them, failed to immediately pick up their crying infants. The anthropologists were adhering to the belief of not spoiling an infant by picking it up every time it cries (Kempe \& Helfer, 1980).

Another related consideration in defining abuse is identifying the distinction between strict or severe discipline and what is abusive. This is highly subjective, and has yet to be resolved. Again, the amount of violence directed towards children that is considered acceptable differs from culture to culture, from time to time (what 
was acceptable as punishment in Victorian times in the United States is no longer acceptable), as well as from house to house (Kadushin \& Martin, 1981). The legal profession has not been helpful in this regard, as most legal definitions of abuse differ from state to state, are intentionally vague, and do not indicate the "amount" of injury that constitutes abuse (Starr, 1988; Mayhall \& Norgard, 1983). Giovanni and Beccerra (1979, as cited in Starr, 1988) conducted a study on over 2,000 subjects, including lawyers, social workers, pediatricians, police officers, and lay people. They developed 78 pairs of vignettes on 13 categories of acts that might be considered abusive. The pairs differed as to the outcome, i.e., in one the child suffered a concussion as a result of being hit with a stick while in the corresponding vignette, no ill-effects were suffered. Each respondent rated 60 randomly selected vignettes on an arbitrary 9-point scale as to their seriousness. The results showed greater agreement the more severe the injury suffered. There was no difference among the different professions on the more severe injuries. Giovanni and Beccerra concluded that there is a need for greater specificity of the nature and extent of the maltreatment on reporting forms.

Although many studies indicate that different sections of the general public hold differing definitions of what is abusive; there exists a core consensus of what minimaliy acceptable care is (Kadushin \& Martin, 1981). Most people 
agree that physical abuse can be defined as "nonaccidental physical harm to the child inflicted by persons responsible for the child's care (Mayhall \& Norgard, 1983; Kadushin \& Martin, 1981). Some people modify this to include only when serious physical harm results.

For the purposes of this study, physical abuse was based on the definition above. Any incident or incidents in which the child came to physical harm by the intentional act of a caregiver, i.e., babysitter, parent, step-parent, or family friend, and the action(s) was intended to cause harm or injury to the child. History of Sexual Abuse

Sexual abuse has also only recently garnered wide spread attention. Prevalence rates of sexual abuse are even more difficult to establish because of the secrecy, shame, and lack of overt physical signs (i.e., bruises, as there may be in physical abuse). Recent studies have indicated that approximately one fifth to one third of all adult women have experienced sexual abuse sometime during their childhood or adolescence (Briere \& Runtz, 1986). However, the prevalence rates depend on the definition of sexual abuse used (Wyatt \& Peters, 1986). Due to this, it is necessary to develop an operational definition of sexual abuse as well.

Definition of Sexual Abuse

Many researchers make distinctions between incest and nonfamilial sexual abuse. Sedney and Brooks (1984) 
discovered that their nonclinical sample of adult women who had experienced incest were more likely to exhibit problems and have adjustment difficulties than women who had experienced nonfamilial abuse. Thus, some authors have decided to look only at the effects of incest, while ignoring extrafamilial abuse (Russell, 1986; Husain \& Chapel, 1983; Courtois, 1979). A second distinction is often made between contact and noncontact behaviors. Contact abuse includes sexual behaviors which involve sexual contact, i.e., fondling, rubbing of genitals against the victim's body, attempted or completed vaginal intercourse, oral sex, and anal sex. Non-contact abuse refers to sexual behaviors that do not require physical contact between perpetrator and victim, i.e., exposure of genitals and solicitations to engage in sexual contact. Some authors have limited the scope of their papers to include only contact abuse (Wyatt \& Peters, 1986; Briere \& Runtz, 1986, Husain \& Chapel, 1983; Sansonnet-Hayden, Haley, Marriage, \& Fine, 1986; Russell, 1983, as cited in Wyatt \& Peters, 1986), while others, Wyatt (1985) and Finkelhor (1979) included both types. Obviously the rates of sexual abuse are going to differ in these two sets of studies.

The age of the perpetrator and victim at the time of the assault has also been an issue. Some early maturing adolescents are able to engage in consensual sexual relationships, and therefore, researchers have tried to 
allow for this factor in their definitions. Finkelhor (1979) used age as the criterion for abuse; he determined that for adolescent victims the perpetrator must be 5 years older than the victim, so all sexual contact by age peers was excluded from his study. Russell (1983, as cited in Wyatt \& Peters, 1986) on the other hand, included all sexual contact with age peers. Wyatt (1985) compromised between the two extremes, setting age as the primary defining characteristic, i.e., 5 years, but considered the experience abusive only if the experience was unwanted by the victim.

For the purposes of this paper, Wyatt's (1985) definition was adhered to, as it appears to be one of the most well thought through and researched. Both contact and non-contact abuse will be included, as well as incestual and extrafamilial types. For extrafamilial abuse of adolescents, the perpetrator must be 5 years older than the victim, or the sexual experience was not wanted and involved some degree of coercion (Wyatt, 1985). The definition of intrafamilial abuse remains the same regardless of the age of the victim.

Abuse and Adults

The research literature (Carmen et al., 1984; Green, 1978 ) indicates a connection between childhood traumas, such as physical and sexual abuse, and the development of many dysfunctional behaviors during later childhood, adolescence, and adulthood. Abuse, especially sexual 
abuse, appears to be related to more suicidal thinking and behaviors, feelings of shamefulness, guilt, depression, anger, impaired impulse control, an inability to trust, and poor problem solving skills (Beck, van der Kolk, 1987; Carmen et al., 1984; Sedney \& Brooks, 1984; Curtois, 1979; Green, 1978).

Beck and van der Kolk (1987) found that $46 \%$ of their adult female patients chronically institutionalized on a psychiatric unit had experienced childhood incest. They described these abused women as more likely to experience sexual delusions, affective symptoms, substance abuse, and to make threats or aggressive behaviors towards others. Moreover, Carmen, et al. (1984) investigated the relationship between physical and sexual child abuse and subsequent psychiatric illness. They conducted a chart review of 188 adult psychiatric inpatients. About $50 \%$ of their patients experienced some type of abuse and appeared to be more likely to have suicidal histories than nonabused patients.

Furthermore, Bryer, Nelson, Miller, and Krol (1987) in one of the few studies conducted on both physical and sexual abuse determined that the effects were similar. Both groups had similarly inflated scores on various measures of maladaptive functioning and adjustment. Moreover, if the subjects experienced both types of abuse, the effects appeared to be additive. In other words, subjects who had been both physically and sexually abused 
were more likely to demonstrate later adjustment difficulties than people who had experienced only one type of abuse.

Much of the research on abuse has focused on how abuse appears to be related to DSM III-R Axis I disorders such as depression, somatization, psychoses, etc. However, recent investigations have begun to look into the relationship between abuse and dysfunctional personality chracteristics or Axis II disorders, e.g, paranoid, hysterical, borderline personality, etc. Personality difficulties tend to be more pervasive and long-standing than Axis I disorders as they are part of the individual's characteristic way of coping with the world. Axis I or adjustment difficulties are seen as more likely to be temporary or transient difficulties.

Herman, Perry, \& van der Kolk (1989) discovered that their female psychatric inpatients who were diagnosed with Borderline Personality Disorder were more likely to have histories of sexual abuse than a psychiatric control group. This finding indicates that abuse is related to personality functioning and not to adjustment difficulties alone.

Goldwater and Duffy (1990) attempted to identify individuals who had been sexually or physically abused as children on the basis of their personality profiles. Goldwater and Duffy's subjects were 182 adult females who had been hospitalized in a psychiatric hospital and were administered the Minnesota Multiphasic Personality Inventory (MMPI) during their hospitalization. Goldwater 
and Duffy determined that the women who had been abused were more likely to show more personality pathology as measured by the MMPI then nonabused patients. Moreover, Roland, Zelhart, and Dubes (1988) conducted a study using college women as subjects in which they concluded that a set of 63 MMPI items could correctly classify 848 of their subjects as having been either sexually abused or not during childhood. Roland, Zelhart, and Dubes (1989), in a follow-up study, investigated the usefulness of the MMPI for discriminating between sexually abused subjects. They divided 171 college women into three groups based on who the perpetrator of the abuse was. They discovered that the group which had experienced father/stepfather incest as compared to those who had experienced sexual contact with other persons or no sexual contact had several MMPI subscales elevated to clinically significant levels. None of the subscales for the other two groups were so elevated. Therefore, they concluded that when the abuse was perpetrated by a father or father figure, more serious personality difficulties tended to coincide. Abuse and Adolescents

Although most of the abuse research has been conducted on adult women, recent work has begun to focus on the more immediate effects of abuse by studying adolescents. Husain and Chapel (1983) studied 437 adolescent females on an inpatient psychiatric unit. All the girls were hospitalized for "emotional" problems. The authors found 
that 61 or 13.98 of their sample reported an earlier incestous involvement. Plummer, Gispert, Hayden, Robbins, and Seifer (1989) discovered that 418 of their sample of hospitalized adolescent suicide attempters had experienced physical abuse, along with $22 \%$ of a control group composed of ideators and adolescents with no suicidal behavior. The figures were similar for sexual abuse $34 \%$ in the attempters group and 168 of the control group.

Moreover, Sansonnet-Hayden, et al. (1986) studied adolescents, aged 13 to 17 , admitted to a psychiatric hospital to determine the relationship between sexual abuse and later psychopathology: They found that victimized adolescents were significantly more likely to attempt suicide, report more depressive symptoms, schizoid/psychotic symptoms, and more somatic complaints than the non-abused psychiatric control group. Furthermore, Spirito, Stark, Fristad, Hart, and OwensStively (1987) compared adolescent suicide attempters admitted to a pediatric medical unit to a group of adolescents referred for psychiatric services while receiving inpatient medical treatment. Spirito et al. found no differences between the two groups on the basis of abuse and thus, concluded that abuse appears to be related to psychiatric problems in general.

The literature indicates that both adults and adolescents who have been abused are more likely to be diagnosed with later Axis I disorders than nonabused 
individuals and to experience more severe symptoms (Sansonnet-Hayden et al., 1986; Bryer et al., 1987). However, it appears, from the research conducted on adults, as if abuse may also be related to more permanent and pervasive difficulties, such as deficits in personality functioning, i.e., Axis II disorders. While there is preliminary evidence for the success of personality inventories in identifying adults with a history of abuse (Roland, Zelhart, \& Dubes, 1989; Roland, Zelhart, \& Dubes, 1988), researchers have not yet attempted to discriminate between abused and nonabused adolescents on the basis of their personality inventories.

Some studies have found that personality inventories have not been successful in discriminating between suicidal and nonsuicidal adolescents (Clopton, Post, \& Larde, 1983; Spirito, Faust, Myers, \& Bechtel, 1988). However, personality inventories have been successful in discriminating between severely disturbed and less disturbed adolescents (Kashani, Dandoy, Vaidya, Soltys, \& Reid, 1990). Therefore, it is believed that abuse is likely to manifest itself in characteristic personality profiles for adolescents.

The MMPI was chosen for use in this study because it describes both the range and severity of patients' symptoms and also their salient personality features. Moreover, the MMPI is presently the most widley used psychological test. It is utilized in a variety of medical settings and is 
universally accepted as an assessment tool for psychiatric patients (Goldwater \& Duffy, 1990). Due to the development of adolescent norms in the 1970's the MMPI is applicable not only with adults but with adolescents as well (Archer, 1987 ).

Family Adjustment

Not only does a history of abuse appear to be related to later adjustment problems, but poor family functioning also has been connected to later difficulties. The literature indicates that children who perceive their families as less supportive are more likely to experience later difficulties or more severe problems than children who perceive their families as more supportive (Asarnow \& Carlson, 1988; Neiger \& Hopkins, 1988; Spirito, Overholser, \& Stark, 1988). Asarnow and Carlson (1988) examined the relative usefulness of adolescents' perceptions of family support, hopelessness, and depression for classifying subjects as suicidal or nonsuicidal. Their subjects were 25 adolescents, aged 8-13, and included both males and females. They determined that adolescents' perception of family support discriminated between suicide attempters and nonattempters with $88 \%$ accuracy. Moreover, Miller, Kabacoff, Keitner, Epstein, and Bishop (1986) assessed the functioning of families with one member being seen by a psychiatric professional and compared them to a control group of families who were not in counseling. They found that families of patients with major depression, alcohol 
dependence, and adjustment disorder reported greater family dysfuction than nonclinical families as measured by the Family Assessment Device (FAD).

Moreover, abuse and poor family functioning often occur conjointly. Tarter, Hegedus, Winsten, and Alterman (1984) investigated whether juvenile delinquents who had been physically abused as children were more likely than nonabused delinquents to be sexually abused. A greater incidence of sexual abuse was found, and the abused delinquents perceived their families as being more dysfunctional than the non-abused group. Kratcoski (1984) studied 295 adolescents who were referred to juvenile court to determine how family functioning affects intrafamily violent behavior, including child abuse, spouse-to-spouse violence, and sibling-to-sibling violence. He found that the more effectively the family functioned the less likely it was that any type of intrafamily violence would occur. It makes intuitive sense that children with abusive histories rate their families as less supportive than children of non-abusive families, since by definition abusive families experience deficits in relating to one another. Hoagwood and Stewart (1989) conducted a study using 30 sexually abused adolescent psychiatric inpatients and 46 age-matched nonabused controls as subjects in order to determine whether sexually abused children perceived their families as more dysfunctional than nonabused children. Hoagwood and Stewart found that sexually abused 
subjects rated their families as having poorer problemsolving skills, more role confusion, and more general pathological functioning than the nonabused subjects, as measured by the Family Assessment Device. In addition, girls reported greater general dysfunction in their families than did males.

The FAD was also chosen for use in the present study as it is a widely used self-report screening device for measuring family functioning. It is applicable for both inpatient and out-patient populations. Moreover, the dimensions measured by it are clinically relevant to this study (Hoágwood \& Stewart; 1989).

It appears from the literature that abused children and children who perceive their families as poorly functioning are in danger of experiencing serious adjustment difficulties during later adolescence. Adults who have experienced prior abuse also appear to have various personality difficulties. It is believed that these personality problems are likely to originate during childhood. Therefore, it is important to identify adolescents who have been abused and who are experiencing difficulties at home, so that treatments which ameliorate the more immediate consequences of these conditions may be administered, as otherwise many abused children may enter adulthood with significant personality dysfunction leading to many maladaptive behavioral and relationship patterns. 
On the basis of the literature, it was believed that adolescents who experienced physical or sexual abuse during earlier childhood, would possess significantly more personality pathology, as measured by the MMPI, than nonabused adolescents. Moreover, adolescents who had experienced both types of abuse would be more symptomatic than those adolescents who experienced only one type of abuse (Bryer et al., 1987). In addition, sexually abused adolescents who were abused by a family member would exhibit more pathological MMPI profiles than adolescents who were abused by a nonfamily member.

It was also predicted that abused adolescents would report more family dysfuction than nonabused adolescents and that adolescents who rated their families as more dysfunctional would have more pathological MMPI profiles than adolescents who perceived their familis as more functional. Finally, abused adolescents could be identified on the basis of their personality profiles and their perception of family functioning as they experienced more pathology.

Method

Subjects

Subjects were between the ages of 13 and 18, and were drawn from consecutive admissions to the adolescent inpatient service of a private child psychiatric hospital in Southeastern New England from January 1, 1989 to October 31, 1992. Past samples have represented all social 
classes, consisted mainly of white females, with a mean age of 15, and indicated that about 30-40\% experience some form of abuse. Hollingshead's (1975) Four Factor Index of Social Status, age, and sex of each subject was tabulated to determine the representativeness of the present sample.

Adolescents were considered abused if they had a notation to that effect in their chart, e.g., the Department of Children, Youth, and Families substantiated an abuse claim or a clinician accepted the allegation. Control subjects were adolescents who had no history of abuse noted in their charts. Charts in which abuse is suspected but not substantiated were not included in the analyses.

\section{Measures}

Epstein, Baldwin, and Bishop's (1982) Family Assessment Device (FAD) is a 60-item self-report questionnaire designed to assess six dimensions of family functioning. Family members are asked to rate how well each item describes their family's functioning on a four point (strongly agree to strongly disagree) scale. The FAD was designed to assess the six dimensions of the McMaster Model of Family Functioning, as well as overall level of family functioning (Kabacoff, Miller, Bishop, Epstein, \& Keitner, 1990). The seven subscales of the FAD include: Problem Solving (PS), Communication (CM), Roles (RL), Affective Responsiveness (AR), Affective Involvement (AI), 
Behavior Control (BC), and General Functioning (GF) (Epstein, Baldwin, \& Bishop, 1983).

The FAD has been shown to have adequate internal consistency (correlational coefficients ranging from .72 to $.92)$, test-retest reliability (.66 to .76), and moderate correlations with other measures of family functioning, e.g., Family Unit Inventory (above .50), and the LockeWallace Marital Satisfaction scale $(r=.53)$. The FAD has also been shown to discriminate between clinical and nonclinical families (Kabacoff et al., 1990; Keitner, Miller, Epstein, \& Bishop, 1986; Epstein, et al., 1983). The FAD has also been able to discriminate between clinical families, in which sexual abuse has occured and clinical families who have not experienced abuse, on several dimensions (Hoagwood \& Stewart, 1989). Moreover, social desirability does not appear to exert a strong influence on FAD scores as correlations between FAD subscales and social desirablity were low (.15 or below) (Byles, Byrne, Boyle, Offord, 1988; Miller, et al., 1986). The hypothesized factor structure was also supported through confirmatory factor analysis, $90 \%$ of the items loaded on the factors hypothesized by the McMaster model.

The Minnesota Multiphasic Personality Inventory (MMPI)

is a 566 item self-report questionnaire designed for use with a clinical population. A fifth grade reading level is required. The respondent answers either "true" or "false" to items which range widely in content, covering 11 
clinical scales and 3 validity scales. The clinical scales include: 1. Hypochondriasis (Hs), 2. Depression (D), 3. Hysteria (Hy), 4. Psychopathic deviance (Pd), 5. Masculinity-Feminity (Mf), 6. Paranoia (Pa), 7. Psychasthenia (Pt), 8. Schizophrenia (Sc), 9. Hypomania (Ma), 0. Social Introversion (Si), and the MacAndrew Addiction Scale (Mac).

The 3 validity scales are not concerned with validity in the technical sense. They represent checks on carelessness, misunderstanding, malingering, and the operation of special response sets and test-taking attitudes. The validity scales include: 1. The Lie Scale (L), 2. The Validity Scale (F), and 3. The Correction Scale, $(K)$. See Table 1 .

Scores are reported in the form of standard scores with a mean of 50 and a standard deviation of 10 , called $T$ scores. These standard scores are used in plotting profiles. Any score falling 2 standard deviations above the mean ( 70 ) is generally taken as the cutoff point for the identification of pathological deviations. It is important to note that the clinical significance of the same score may differ from one scale to another.

Norms were developed for adolescents by Marks, Seeman, and Haller in 1974, and are still widely used today. They are based on 1,800 normal adolescents, and over 1200 adolescents between the ages of 12 and 18 who were recieving some type of psychiatric treatment. The norms 
Table 1. Descriptions of the Minnesota Multiphasic Personality Inventory Standard Scales.

\begin{tabular}{|c|c|c|c|}
\hline & $\begin{array}{l}\text { Scale Name } \\
\text { Lie }\end{array}$ & $\begin{array}{l}\text { Abbrev. } \\
\quad(\mathrm{L})\end{array}$ & $\begin{array}{l}\text { Description } \\
\text { Socially correct behavior }\end{array}$ \\
\hline & Validity & $(\mathrm{F})$ & $\begin{array}{l}\text { How much one's thoughts } \\
\text { differ from population }\end{array}$ \\
\hline & Correction & $(\mathrm{K})$ & Defensiveness and guardedness \\
\hline 1 & Hypochondriasis & (Hs) & Number of bodily complaints \\
\hline 2 & Depression & (D) & Mood, sadness, depression \\
\hline 3 & Hysteria & (Hy) & $\begin{array}{l}\text { Propensity to act self- } \\
\text { centered, dramatic, anxious }\end{array}$ \\
\hline 4 & Psychapthc Deviance & $(P d)$ & $\begin{array}{l}\text { assertiveness, hostility } \\
\text { towards authority }\end{array}$ \\
\hline 5 & Masc.-Feminitiy & (Mf) & $\begin{array}{r}\text { identification w/ sex role, } \\
\text { passiveness, rigidity }\end{array}$ \\
\hline 6 & Paranoia & $(\mathrm{Pa})$ & $\begin{array}{c}\text { interpersonal sensitivity, } \\
\text { suspiciousness }\end{array}$ \\
\hline 7 & Psychasthenia & $(P t)$ & anxiety, self-confidence \\
\hline 8 & Schizophrenia & (Sc) & $\begin{array}{l}\text { mental confusion, logical } \\
\text { thinking }\end{array}$ \\
\hline 9 & Hypomania & $(\mathrm{Ma})$ & psychic (mental) energy \\
\hline & Social Isolation & $(\mathrm{Si})$ & $\begin{array}{l}\text { preference for being alone } \\
\text { or with others }\end{array}$ \\
\hline
\end{tabular}

(Archer, 1987; Duckworth \& Anderson, 1986) 
are reported separately for males and females at age groupings of $17,16,15$, and a category of 14 and below. Moreover, Williams and Butcher (1989) determined that using the same MMPI scale descriptors for adolescents that are used for adults is empirically valid.

The research that has been done using adolescents as subjects has found that the MMPI has been useful in discriminating between delinquents and nonsymptomatic individuals as well emotionally disturbed adults and nonsymptomatic adults. Much research has been conducted using the MMPI as an indicator of differential diagnosis with some success (Archer; 1987; Dahlstrom, Welsh, \& Dahlstrom, 1975). In addition, Archer (1987) studied both adult and adolescent inpatients and determined that the MMPI profiles of adolescents were not as extreme as those of adults. He suggested that instead of using a score of 70, which is the generally accepted cut-off score above which indicates the presence of psychopathology for adults, but that a score of 65 be substituted as defining clinical range elevations for inpatient adolescents. Archer (1987) further stated that relying on a rigid cut-off score to define clinically significant elevations did not appear to be supported with adolescents, and that the MMPI would prove to be much more useful to the clinician if he/she used flexibility when defining clinical symptomatology. Archer found that $26.6 \%$ of adolescent inpatients had normal or nonpathological MMPI profiles (did not score 70 or above 
on any clinical scale). However, when he lowered the criterion score to 65 , the percentage of "normal" profiles was reduced to 118 .

Test-retest coefficients with an eight-month interval between tests range from .42 to .76 for college students, .36 to 61 for adolescents with a three year interval, and .35 to .76 for female psychiatric patients with a one year interval. In general, the longer the intertest interval the lower the correlation coefficient (Dahlstrom et al., 1975). Internal consistency coefficients are also adequate, with most scores falling above .80 .

Hollingshead's (1975)' Four Factor Index of Social

Status is based on the education, occupation, sex, and marital status of each subject's parents. A combination of this information is used to arrive at a social ranking from 1 (unskilled laborer) to 5 (major business professional). The parent with the highest education or occupational level is used if parents belong to different rankings. Construct validity has been demonstrated for each of the scales by correlations with data taken from the 1970 United States Census, such as the mean years of school completed with education scale (males=.835, females=.849), and the mean income earned with the occupation scale (males=.781, females=.672). Although final validation information for the relationship between marital status and social status was not available, preliminary factor analysis studies indicate a meaningful correspondence. 


\section{Procedure}

An archival study was conducted. Psychological charts were reviewed in which MMPI and FAD protocols were kept, if they had been administered. MMPI standard scores, 63 specific MMPI item responses, and the seven scaled scores for the FAD were recorded. Corresponding medical charts for the 541 subjects who had either an MMPI or FAD protocol in their research chart were then reviewed. Abuse history was recorded, as well as other demographic data, i.e., sex, number of family members, living situation, Hollingshead score, and age.

The MMPI was administered to every adolescent as part of normal admissions protocol within the first 5 days of admission. For adolescents who have been admitted on multiple occasions, only the MMPI administered during the first admission was included. The FAD was completed by 223 respondents during the initial days of admission. The FAD was typically administered on only one of the adolescent units, and therefore, fewer adolescents completed this questionnaire. The MMPI and FAD were scored by a psychology technician. Previous research with this population (Shaunesey, Cohen, Plummer, \& Berman, 1993) has indicated that a thorough screening for abuse is done at time of admission, and therefore, it is unlikely that new cases of abuse would be identified with use of a face-toface interview format. 
Results

The mean age of the 543 individuals comprising the sample was 14.92 years (ranging from 13 to 18 ), with 61.08 of the sample being female and 39.08 were male. The mean family size was 3.96 , suggesting that a family typically consisted of 4 members. Of the entire sample, $82.8 \%$ lived with at least one parent, 4.08 lived with relatives, 4.68 were in foster placements, $7.6 \%$ resided in group homes, and $1.0 \%$ had other living arrangements, e.g., living independently. The mean Hollingshead score was 34.45, indicating that most of the adolescents' parents worked in skilled crafts, clerical, or sales type positions.

of the total sample, $36.8 \%$ were physically abused, $34.7 \%$ experienced sexual abuse, and of these abused subjects, 19.28 experienced both types of abuse. Table 2 indicates the number of males and females in each of the abuse categories: none, both physical and sexual abuse, sexual only, and physical abuse alone. These statistics are similar to those from previous studies (Plummer, et al., 1989; Shaunesey, et al., 1993), indicating that the present sample is representative of the patient population admitted to this hospital.

Of the 193 subjects who were physicaly abused, 27.23\% experienced abuse at the hands of more than one perpetrator. The father or father-figure (e.g., mother's boyfriend, stepfather, etc.) was the initial or primary perpetrator of physical abuse for $63.9 \%$ of the subjects, 
Table 2. Number of Males and Females in each Abuse Group.

\section{TYPES OF ABUSE}

No Abuse Both Sexual Physical

\begin{tabular}{|c|c|c|c|c|c|}
\hline \multirow{3}{*}{ SEX } & Females & 121 & 78 & 66 & 56 \\
\hline & Males & 129 & 23 & 15 & 36 \\
\hline & Total & 250 & 101 & 81 & 92 \\
\hline
\end{tabular}


the mother in 23.08 of the cases, relatives (e.g., brothers, uncles, aunts, etc.) accounted for $4.7 \%$ of the perpetrators, $6.8 \%$ of the subjects were abused by nonrelated acquaintances, and strangers perpetrated $1.6 \%$ of the physical abuse.

For the 182 sexually abused subjects, 26.978 were victimized by multiple perpetrators. The primary or initial perpetrator was again the father or father figure in $38.2 \%$ of the cases, family friends accounted for 29.28 of the abuse, relatives perpetrated $24.7 \%$ of the sexual abuse, strangers $4.5 \%$, and mothers accounted for $3.4 \%$.

In order to see if the effects of physical and sexual abuse differed from each other significantly and to compare males and females on the different scales, a two (sex) by four (abuse: none, physical sexual, both) repeated measures multivariate analysis of variance was conducted, on the 14 separate standardized scales of the MMPI. Significant twoway interactions, Abuse by Scales, $\underline{F}(13,39)=1.64, \underline{p}<.05$ level, and Sex by Scales, $\underline{F}(13,39)=6.07, \underline{p}<.05$ level were found. These findings indicate that individuals in the different abuse categories and of different sexes score differentially on at least some of the MMPI scales.

Simple effects tests of the Abuse $x$ Scales interaction for differences among the four abuse groups for the 14 standardized scales were conducted (See Figure 1). Significant findings resulted for the F Validity Scale; the Pd Scale, the Sc Scale, and the Ma Scale (See Table 3). 


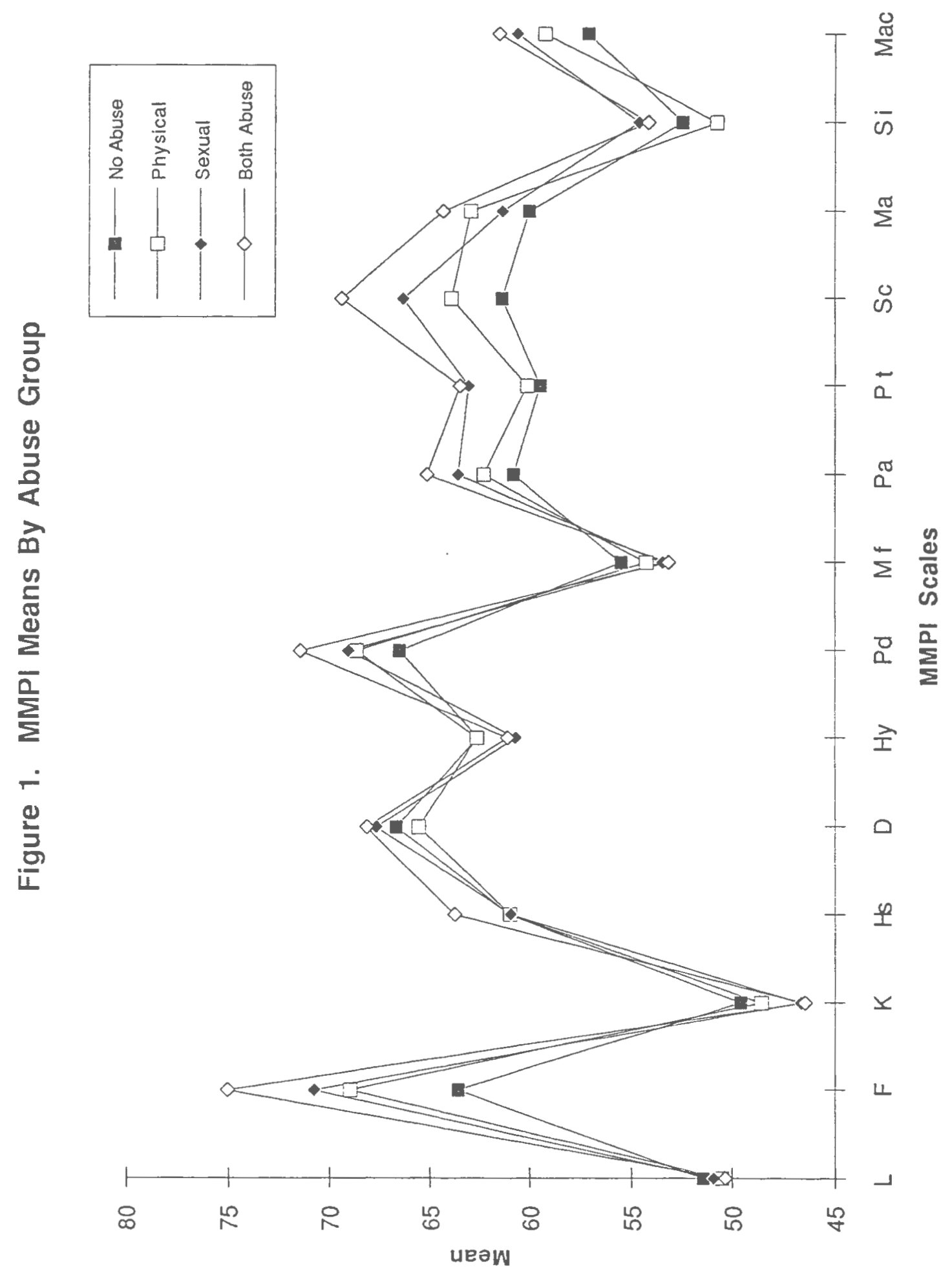


Table 3. Means for the Significant Simple Effects Tests for Differences among the Four Abuse Groups for the 14 MMPI Scales.

Scale
NONE PHY SEX BOTH F Ratio $\begin{gathered}\text { Significant Diff. } \\ \text { Between Groups }\end{gathered}$

F $\quad 63.6168 .97 \quad 70.7875 .037 .58 *$ (Both, Sex) \& (None)

Pd $66.4968 .6369 .04 \quad 71.44 \quad 2.68 * \quad$ (Both) \& (None)

Sc $61.4063 .9366 .3169 .36 \quad 5.06 * \quad$ (Both) \& (None)

Ma $60.0262 .9761 .36 \quad 64.34 \quad 3.26 * \quad$ (Both) \& (None)

d.f. $=3 \& 397$ for all analyses.

* $\mathrm{p}<.05$ level 
Follow-up Tukey tests revealed that adolescents who had experienced both types of abuse $(M=75.03)$ or sexual abuse alone ( $M=70.78)$ scored significantly higher on the $F$ scale than adolescents who had not experienced abuse $(M=63.61)$. In addition, those adolescents who had experienced both types of abuse for the Pd $(M=71.44), \operatorname{Sc}(M=69.36)$, and Ma $(M=64.34)$ Scales scored significantly higher than those who were not abused on each scale respectively ( $M=66.49$, $\mathrm{M}=61.40$, and $\mathrm{M}=60.02$ ).

The sex by Scales interaction was also investigated by simple effects tests in the same manner as above in order to compare males and females on the different MMPI scales. See Table 4 for a listing of significant differences and Figure 2 for a pictorial description. The results indicated that females had signficantly higher scores on the F, D, Pd, Pt, and Sc Scales than males. Females scored signficantly lower on the masculinity-feminity scale than males, however, this is to be expected since the scale is designed so that females endorse a fewer number of items classifying them as more feminine, while males with typical masculine traits usually endorse more items, causing them to score higher, falling in the higher scoring ranges.

A two (sex) by four (abuse category) repeated measures multivariate analysis of variance was conducted on the 7 FAD scales, in order to determine whether males and females in the different abuse cateogries (none, physical, sexual, both) perceived differing amounts of family dysfunction, as 
Table 4. Means for the Significant Simple Effects Tests for Males and Females on the Scaled MMPI Dimensions.

\begin{tabular}{cccc} 
Scale & Females & Males & \multicolumn{1}{c}{ F } \\
F & 70.93 & 63.03 & $16.61 *$ \\
D & 68.53 & 64.34 & $8.02 *$ \\
Pd & 69.61 & 66.01 & $6.95 *$ \\
Mf & 51.31 & 59.61 & $52.07 *$ \\
Pt & 62.27 & 58.86 & $5.11 *$ \\
Sc & 66.19 & 60.94 & $10.23 *$
\end{tabular}

d.f. $=1$ and 399

* $\mathrm{p}<.05$. 


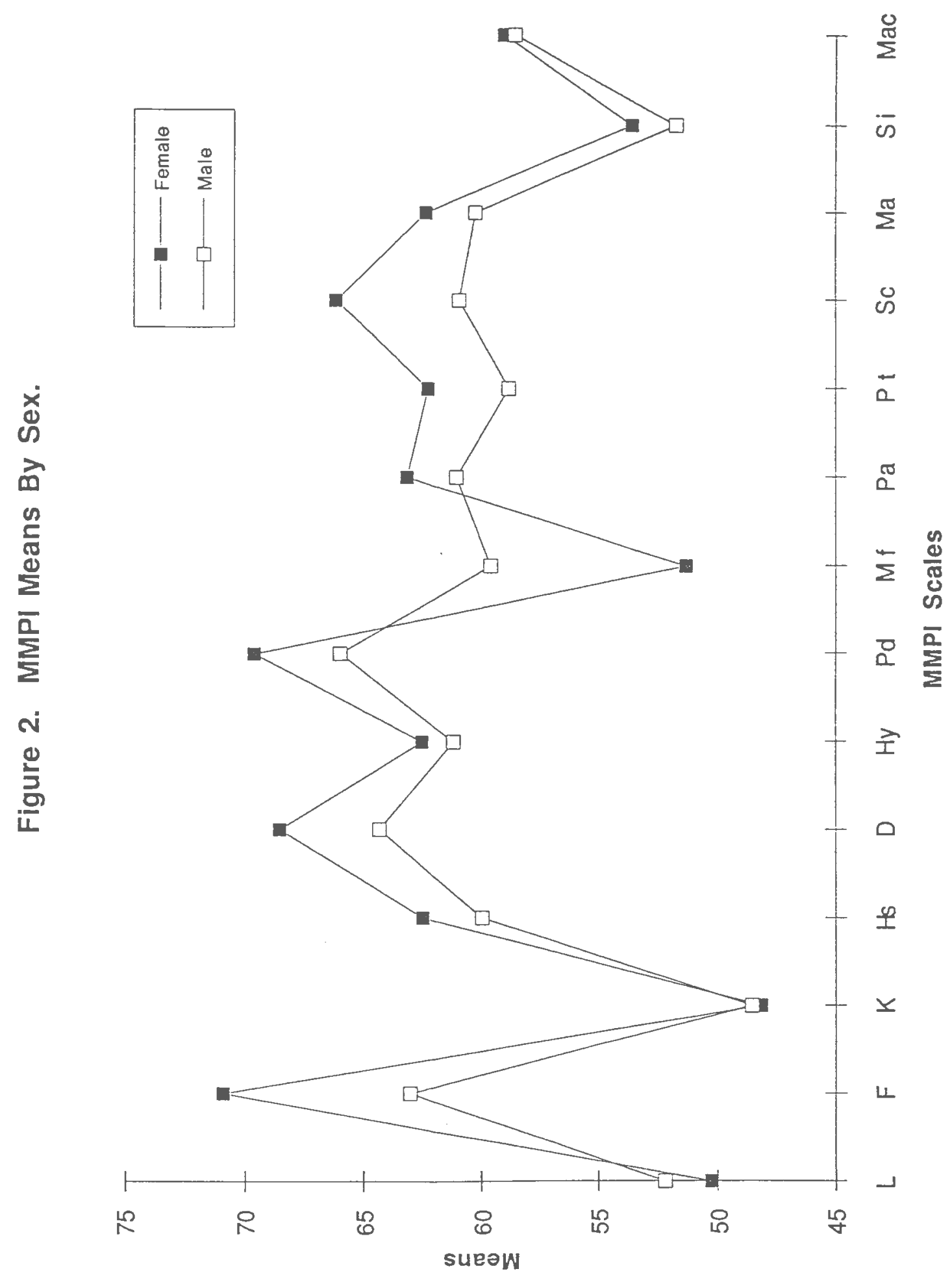


measured by the FAD. No significant differences were found.

In order to compare incest survivors to those individuals who had experienced nonfamilial sexual abuse on the 14 MMPI standardized scales and on the 7 FAD scales, two repeated measures multivariate analyses of variance were conducted. No significant differences were found for the MMPI profiles of incest victims and nonfamilial abuse victims, even when males and females were viewed separately. However, a significant Group (incest victim vs. nonfamilial abuse victim) by scales interaction was found for the FAD, $\underline{F}(6,390)=2.68, \underline{p}<.05$, indicating that incest victims and nonfamilial abuse victims perceive different amounts of family dysfunction on at least some of the dimensions than nonfamilial abuse victims (See Figure 3).

Simple effects tests were used to investigate the Group by Scales interaction for the FAD. Incest victims scored significantly lower on the problem solving $(M=2.18)$, communication $(\mathrm{M}=2.30)$, and general functioning $(\mathrm{M}=2.25)$ scales than nonfamilial abuse victims ( $M=2.56, M=2.63$, and $\mathrm{M}=2.58$, respectively). This finding suggests that incest victims perceive fewer problems along these three dimensions than victims of nonfamilial abuse.

In order to determine the relationship between the scores on the 14 MMPI scales and adolescents' perceptions of their family's functioning, a repeated measures 


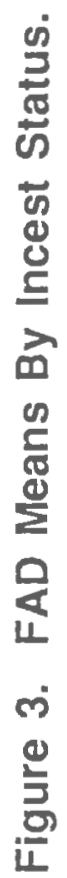

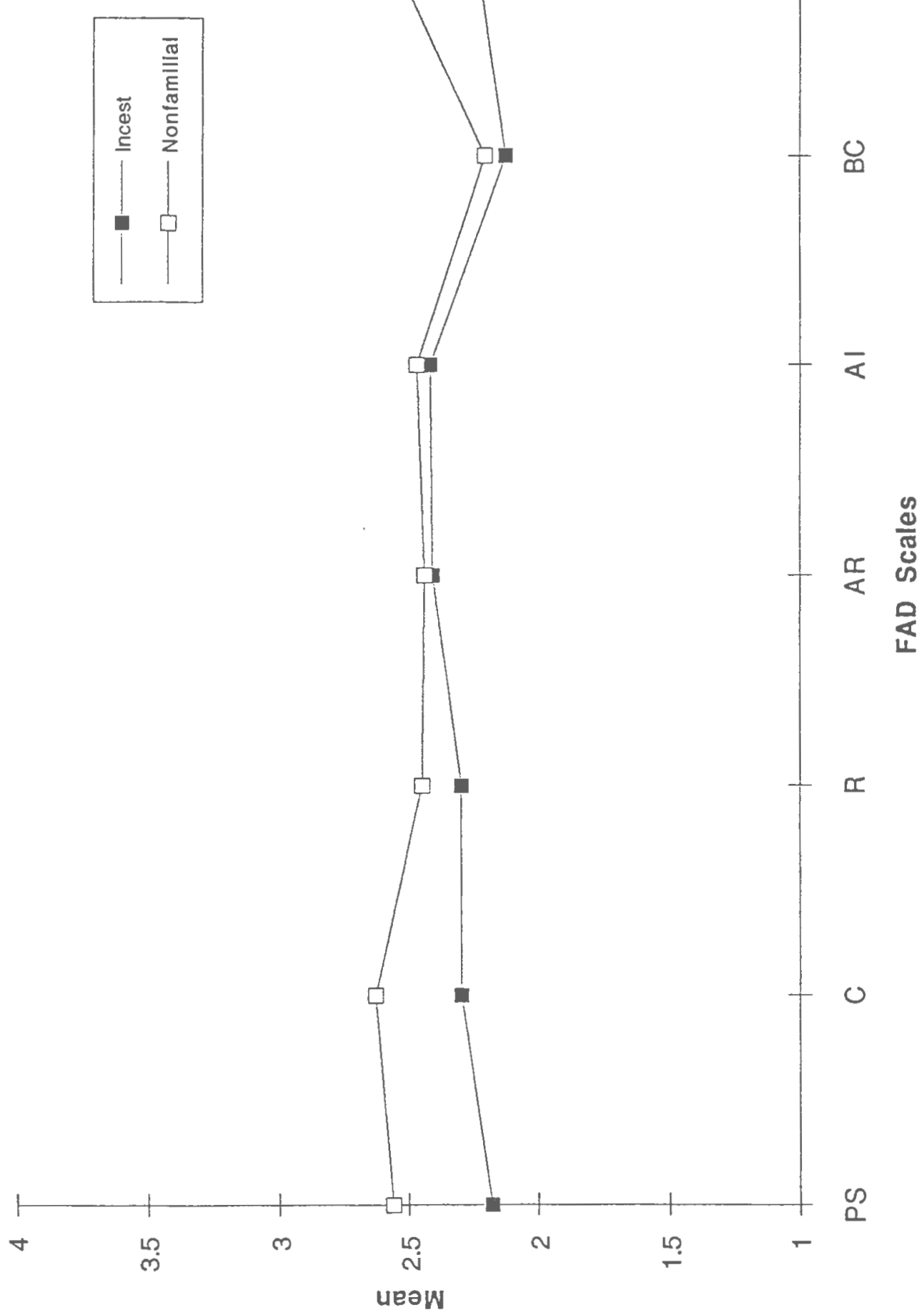


multivariate analysis of variance was conducted. The general functioning scale of the FAD was classified into 3 categories (no-low dysfunction: FAD score less than or equal to 2; medium dysfunction: FAD score greater than 2 and less than or equal to 3 ; and high dysfunction: FAD score greater than 3 ) and was used as the independent variable, with the scaled MMPI scores as the dependent variables. An interaction (FAD by Scales) effect $\underline{F}(13,26)$ $=3.73, \underline{p}<.05$ was found (See Figure 4 ).

Simple effects tests for the FAD by Scales interactions were conducted on each of the 14 MMPI scales to determine on which scales the significant differences in family dysfunction belonged. See Table 5 for a listing of the significant differences. Tukey follow-up tests revealed that in general, the more family dysfunction an adolescent perceived in his or her family, the higher the MMPI score. This trend was reversed for the $L$ and $K$ validity scales, as the more family dysfunction reported the lower the scores on the two scales (however, on the $L$ and $\mathrm{K}$ scales, low scores are also suggestive of dysfunction).

A step-wise discriminant analysis was conducted to determine if 63 MMPI items, which were specifically chosen by Roland et al., 1988, could be used to separate sexually abused females from females who had experienced no sexual abuse on the basis of these items. A significant lineár function was found, Wilks' Lambda $=.6792, \times 2(27)=81.44$, 


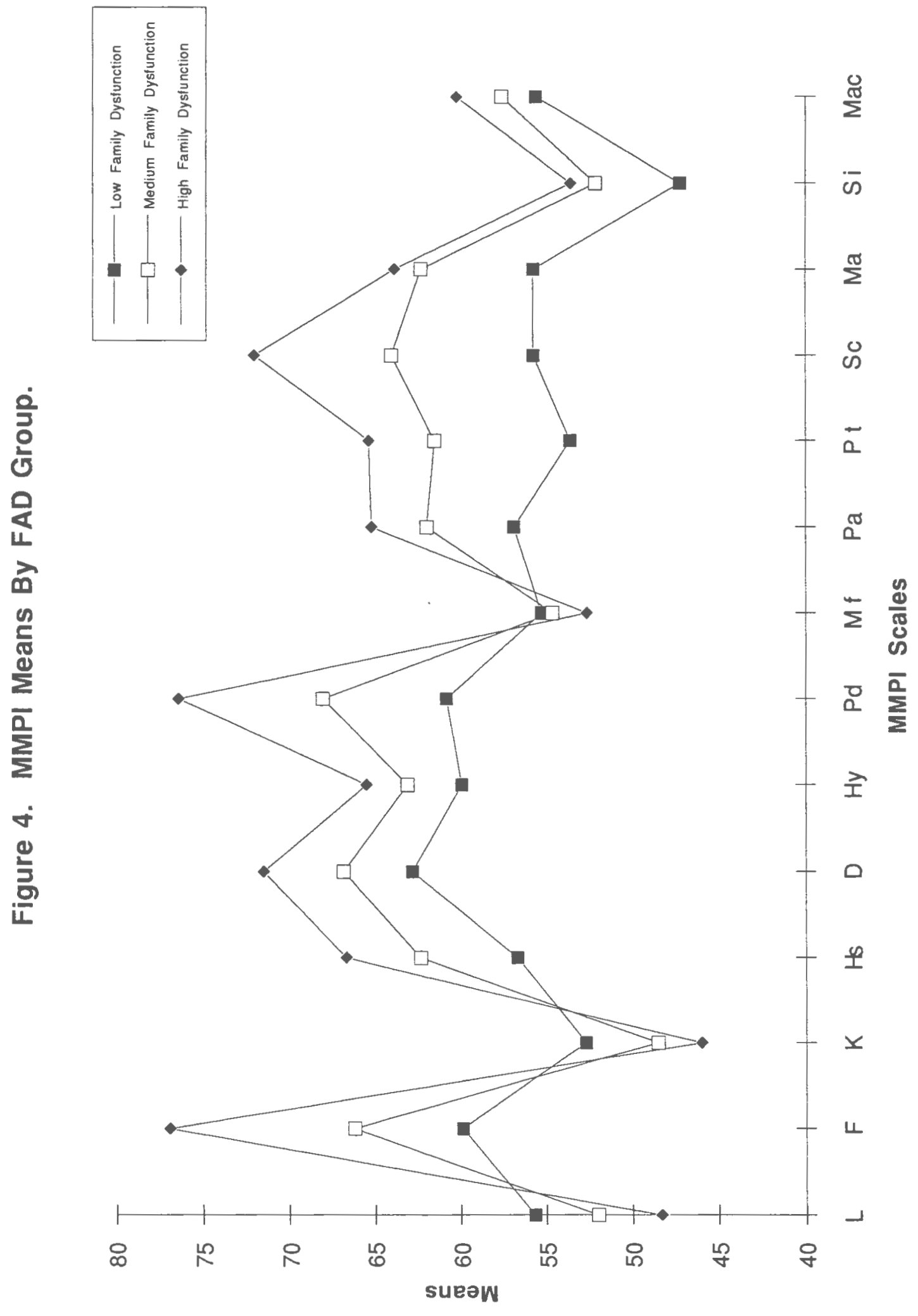


Table 5. Means for the Simple Effects Tests and Tukey Follow-up Tests for 3 FAD Groups on 14 Scaled MMPI Dimensions.

\begin{tabular}{|c|c|c|c|c|c|}
\hline Scale & Low & $\begin{array}{r}\text { Means } \\
\text { Medium }\end{array}$ & High & F Ratio & $\begin{array}{l}\text { Signficant Diff } \\
\text { Between Groups }\end{array}$ \\
\hline $\mathrm{L}$ & 55.68 & 52.00 & 48.33 & $4.00 *$ & $(L) \&(H)$ \\
\hline $\mathrm{F}$ & 59.89 & 66.23 & 76.96 & $6.53 *$ & $(\mathrm{~L}, \mathrm{M}) \&(\mathrm{H})$ \\
\hline K & 52.70 & 48.50 & 46.00 & $4.52 *$ & $(\mathrm{~L}) \&(\mathrm{M}, \mathrm{H})$ \\
\hline $\mathrm{Pd}$ & 60.84 & 68.07 & 76.44 & $12.40 *(\mathrm{~L})$ & $\&(\mathrm{M}, \mathrm{H}),(\mathrm{M}) \&$ \\
\hline $\mathrm{Pa}$ & 56.89 & 61.98 & 65.22 & $5.01 *$ & $(L) \&(M, H)$ \\
\hline Pt & 53.57 & 61.51 & 65.37 & $6.25 *$ & $(\mathrm{~L}) \&(\mathrm{M}, \mathrm{H})$ \\
\hline Sc & 55.72 & 64.03 & 72.04 & $8.76 *$ & $(L) \&(M, H)$ \\
\hline Ma & 55.75 & 62.31 & 63.85 & $6.65 *$ & $(\mathrm{~L}) \&(\mathrm{M}, \mathrm{H})$ \\
\hline
\end{tabular}

d.f. $=2 \& 188$ for all analyses.

$* \underline{p}<.05$ level 
$\mathrm{p}<.05$, using 27 of the 63 items, which correctly classified $75.30 \%$ of the 140 cases in the no abuse group, and 107 in the sexual abuse group (See Table 6).

of the 27 items which were included in the significant discriminant function, the sexually abused subjects endorsed items more often than nonabused subjects, which suggested that sexually abused females experience more problems with self-esteem, self-efficacy, social skills, feelings of inadequacy, isolation, family dysfunction, feelings of being different and damaged, and negative reactions towards sexuality.

Roland et al., 1988 also determined that 29 (i.e., the minimum number of items in the subscale scored in the direction specified) was the cut-off score which classified subjects who reported abuse. An analysis of variance with sexual abuse (yes/no) as the independent variable and the total score of the 63 chosen MMPI items as the dependent variable was conducted. A signficant main effect for sexual abuse was found, $\underline{F}(1,224)=4.65$, $\underline{p}<.05$ level, indicating that nonsexually abused subjects ( $M=30.78)$ scored significantly lower than the sexually abused subjects $(M=35.09)$.

Another step-wise discriminant analysis was conducted to determine if the 63 items chosen by Roland et al., 1988, could correctly classify both male and female subjects into one of the four abuse cateogries: no abuse, physical abuse, 
Table 6. Standardized Canonical Discriminant Function Coefficients for the 27 Selected Items of the MMPI used to Classify Sexually and Nonsexually Abused Females and Group Centroids for the Signficant Linear Discriminant Function.

$\begin{array}{rr}\text { MMPI\# } & \text { Standard Canonical Coefficients } \\ 418 & .468 \\ 64 & -.427 \\ 315 & .399 \\ 21 & -.393 \\ 168 & .389 \\ 368 & -.384 \\ 142 & .369 \\ 284 & -.350 \\ 416 & -.308 \\ 238 & .296 \\ 470 & .280 \\ 267 & .279 \\ 282 & .267 \\ 278 & .266 \\ 544 & -.265 \\ 361 & .255 \\ 299 & .235 \\ 62 & .228 \\ 345 & -.224 \\ 431 & -.221 \\ 526 & -.212 \\ 549 & -.206 \\ 86 & -.200 \\ 336 & -.195 \\ 236 & .193 \\ 129 & .189 \\ 67 & -.158\end{array}$

\begin{tabular}{lccc} 
& \multicolumn{2}{c}{ Category } & \\
& NO ABUSE & SEXUAL & ABUSE \\
Group & -.588 & .796
\end{tabular}


sexual abuse, physical abuse, both types. Three canonical discriminant functions were found, although only the first two were significant, Wilks' Lambda $=.5602, \times 2(93)=$ 201.97, and Wilks' Lambda $=.7706, \times 2(60)=90.80, \mathrm{p}<.05$.

of the 63 MMPI items, 31 were selected for inclusion in the two significant linear functions. See Table 7 for a description of the canonical coefficients and group centroids for both significant linear functions. The linear functions correctly classified 52.518 of the 432 subjects into one of the four abuse categories.

An analysis of variance with abuse category as the independent variable and the total of the 63 MMPI items as the dependent variable was conducted in order to determine whether the groups could be distinguished on the basis of their total score. A significant main effect for abuse was found, $\underline{F}(3,363)=3.62, \underline{p}<.05$, indicating that the type of abuse experienced is related to the number of items answered in a positive direction.

Tukey follow-up tests indicated that the group which experienced both types of abuse $(M=35.49)$ scored significantly higher than the no abuse group ( $M=29.32)$, although the both group did not differ significantly from either the physical abuse $(M=30.48)$ group, or the sexual abuse $(M=33.29)$ group.

Next, in order to determine whether individuals' scores on the 14 MMPI scales could correctly classify them 
Table 7. Standardized Canonical Discriminant Function Coefficients for the 31 Selected Items of the MMPI used to Classify Subjects into Abuse Category, and Group Centroids for the Two Significant Linear Functions.

MMPI
315
470
158
61
168
361
284
67
62
217
418
282
336
549
368
21
356
186
64
526
308
47
43
328
342
182
366
398
416
41
238

Standardized Coefficients Function 1

.393

.389

.387

.318

.316

.302

$-.279$

$-.274$

.273

$-.269$

.265

.242

$-.241$

$-.234$

$-.230$

$-.217$

.196

$-.174$

$-.162$

$-.157$

.134

$-.128$

.118

$-.110$

.105

.087

.018

.017

$-.009$

.006

.004
Function 2

$-.220$

.035

.098

.233

$-.213$

$-.213$

$-.037$

$-.110$

.261

$-.356$

$-.017$

.102

$-.016$

$-.243$

$-.007$

$-.156$

$-.105$

.315

.501

.345

.404

$-.341$

$-.268$

$-.300$

.317

.303

$-.369$

$-.083$

.279

.446

.127
Group

Centroids

FUNCTION 1 FUNCTION 2
Category

NO ABUSE BOTH SEXUAL ONLY PHYSICAL ONLY $-.477 \quad .516$

$-.261$
1.21

$-.239$
$-.181$ .881 
into one of the four abuse categories (none, physical, sexual, both), a stepwise discriminant analysis was conducted, using the scores on each of the 14 standardized scales to predict which abuse group each subject belonged. Three canonical discriminant functions were formed, however, only the first was significant (Wilks' Lambda = $.8979, \times 2(12)=42.65 . \mathrm{p}<.05)$. The significant linear function included four of the 14 variables: the F, Hy, Si, and Mf scales (See Table 8). Using the discriminant analysis function, subjects were classified on the basis of their 14 MMPI scores. In the original comparison, there were 245 no abuse subjects, 97 both types, 87 physical, and 80 sexual only. Of these, $40.28 \%$ were correctly classified.

Finally, in order to determine whether any of the repondents were actually scoring within the pathological range on the MMPI and FAD, protocols were classified as either healthy or unhealthy, based on clinically defined cutoff scores. For the MMPI validity scales, scores above 55,70 , and less than 45 or above 60 , respectively, were considered as indicative of emotional problems. For the MMPI clinical scales, a score above 70 was considered unhealthy. For the FAD, scores above 2.2, 2.2, 2.3, 2.2, $2.2,1.9$, and 2.0 , respectively, were considered clinically significant. Two (healthy vs, unhealthy) by four (abuse cateogry: none, both, sexual only, physical only) chi square analyses were then conducted. For the MMPI, 
Table 8. Standardized Canonical Discriminant Function Coefficients for the 4 MMPI Standard Scales used to Classify Subjects into Abuse Group, and Group Centroids for the Significant Linear Discriminant Function.

$\begin{array}{cc}\text { Scale } & \begin{array}{c}\text { Standardized } \\ \text { Canonical Coefficients }\end{array} \\ \text { F } & 1.11 \\ \text { Hy } & -.591 \\ \text { Mf } & -.340 \\ \text { Si } & -.140\end{array}$

Group

Category

NO BOTH SEXUAL PHYSICAL

$\begin{array}{lllll}\text { Centroids } & -.304 & .486 & .240 & .063\end{array}$ 
significant relationships between emotional health and abuse category were found for several scales: F, $K, P t$, and Sc (See Table 9). No significant relationships were found for any of the FAD scales.

However, Archer (1987) stated that 65 may be a more appropriate cut-off point for defining clinical elevations on the MMPI for adolescents than a score of 70 . Therefore, the two (pathology) by four (abuse group) chi square analyses were recomputed using 65 as the cut-off point for defining clinical significance. Significant relationships between abuse category and pathology were found for the F, $\mathrm{K}, \mathrm{Pa}$, and Sc Scales. See Table 10.

\section{Discussion}

The literature suggests that physical and sexual abuse are related to later difficulties in both adolescent and adult functioning (Beck \& van der Kolk, 1987; Bryer, 1987; Carmen et al., 1984; Sedney \& Brooks, 1984; Meiselman, 1980: Curtois, 1979, and Green, 1978). Recent studies have begun to focus on the nature of these difficulties, investigating whether the individual's problems are transient, adjustment type disorders (Axis I) or more permanent and pervasive, character disorders (Axis II), often finding that abuse victims do appear to suffer from personality disorders (Tsai, Feldman-Summers, \& Edgar, 1979; Roland, et al., 1988; Scott \& Flowers, 1988; Herman, et al., 1989, and Goldwater \& Duffy, 1990). However, few studies have examined the relationship between abuse and 
personality functioning in adolescents, although preliminary results have suggested the existence of a connection between character disorders and abuse (Scott \& Stone, 1986; Scott \& Stone, 1986a).

The results of the present study provide support for the findings of previous studies which found that abused subjects demonstrated more difficulties in personality functioning, as measured by the MMPI, than nonabused individuals. In the present investigation, being abused, both physically and sexually, appeared to be related to a more elevated MMPI profile than being sexually or physically abused alone or not being abused at all, although sexual abuse alone was also related to elevated scores on one scale of the MMPI. Specifically, the adolescents who had experienced both types of abuse and the sexually abused alone group scored significantly higher on the F validity Scale of the MMPI than the physical and no abuse groups, suggesting that both these groups were experiencing unusual or markedly unconventional thinking than the other two groups. Elevations on this scale have also been interpreted as "cries for help" or that the individuals are in extreme distress.

Moreover, the hospitalized adolescents who had experienced both physical and sexual abuse scored significantly higher on the Pd, Sc, and Ma Clinical Scales of the MMPI than the hospitalized individuals who had not experienced abuse or who had only experienced one type of 
abuse. In other words, the physically and sexually abused adolescents scored higher on dimensions which measured hostility towards authority, disorganized thinking, and psychic energy than the other three groups. Furthermore, individuals with elevations on these three scales often have histories of aggression (Duckworth \& Anderson, 1986). These findings provide additional evidence that a history of being both physically and sexually abused may, in fact, lead to more difficulties in personality functioning, although, it does not appear that experiencing one type of abuse differentiates abused from nonabused psychiatric inpatients, as measured by the clinical scales of the MMPI.

In looking at the Group by Scales interaction it appears that there was also a trend for the adolescents who had experienced both types of abuse to have the highest mean profile, the sexual abuse group had the second highest mean profile, followed by the mean of the physical abuse group, and the no abuse group with the lowest mean profile. These findings also indicate that being exposed to both types of abuse may be related to more severe difficulties than either physical or sexual abuse alone. The results of this investigation are consistent with previous studies which found that the effects of abuse were additive in that they were related to more difficulties in functioning than the presence of just one type of abuse (Shaunesey, et al., 1993; Bryer et al., 1987), as the presence of both physical 
and sexual abuse were related to higher scores on the MMPI than either type of abuse alone or no abuse.

The present study did not find significant differences between adolescents who had experienced one type of abuse and the no abuse group. This finding is consistent with some other authors, such as Tarter, et al., (1984) who found no differences in the MMPI profiles of physically abused and nonabused delinquents who were in a residential treatment program. Moreover, Tarter et al.'s sample included more male than female adolescent subjects. Goldwater and Duffy (1990) also found that adult inpatients, who had experienced either sexual or physical abuse, had a similar, but overall more elevated mean MMPI profile when compared to a physically abused inpatient control group's.

Again, the findings of these studies suggest that the presence of both types of abuse are necessary in order to obtain significant differences when compared to inpatient controls. Many previous studies only recorded incidents of sexual abuse and did not ask respondents about physical abuse history. Therefore, some previous studies such as Scott \& Stone's 1986 investigation may actually have included subjects who were physically and sexually abused, not just sexually victimized, which would explain why the sexual abuse alone group in this study was not differentiated from the physical and no abuse groups on any of the Clinical MMPI Scales. 
However, not only were the victims of sexual abuse alone not differentiated, for the most part, from the physical abuse and nonabused groups, but the present study did not provide support for the belief that incest victims experience more difficulties in personality functioning than victims of nonfamilial sexual abuse, as other researchers such as Roland et al. (1989) have found. There were no differences between the MMPI profiles of nonfamilial and familial abuse victims, suggesting that both groups were experiencing similar difficulties in personality functioning .

The finding in the present study that incest and nonfamilial abuse victims did not differ on the MMPI may be due to the fact that in the present study all subjects were hospitalized, indicating that all groups included in the study were experiencing a great deal of emotional stress at the time they filled out the questionnaires. In contrast, many of the previous studies used outpatient and nonclinical samples. Thus, if nonclinical samples had been used in the present investigation, differences between the physical, sexual, and no abuse groups may have been noted, as in other studies which have used outpatient or nonclinical populations (Roland et al., 1989; Scott and Stone, 1986; Scott \& Stone, 1986a, and Tsai at al., 1979). In addition, subjects were classified as incest victims if the perpetrator of the abuse was the natural father, step-father, mother's boyfriend, or another male 
relative. This is a broader definition than most other studies have used. Typically, incest victims have been defined in the literature as having been abused by a natural, adopted, or step-father (Roland et al., 1989; Scott \& Stone, 1986; Scott \& Stone 1986a). Therefore, the present study found no differences between incest and nonfamilial abuse victims, as in other studies, because the incest group may have been too heterogeneous.

However, according to the Group by Scales interaction which was found when incest and nonfamilial abuse victims were compared on the FAD, there were differences between the incest and nonfamilial abuse victims' perceptions of the way their families function. Victims of incest rated their families as healthier than adolescents who had experienced nonfamilial abuse on three of the FAD's dimensions: problem solving, communication, and general functioning. These results are surprising in that they are in direct opposition to what researchers have found in the past (Hoagwood \& Stewart, 1989). The literature has indicated that incest families typically have poorer overall functioning than nonfamilial abuse families, especially in the areas of communication, roles, and general functioning. However, it has also been demonstrated in the literature that incest families are very invested in maintaining the appearance of normalcy to outsiders (Finkelhor, 1979; Sgroi, 1982). Thus, this study may have actually measured the adolescent's desire to 
support their parents' illusion of normalcy to hospital personnel.

Moreover, the FAD measures the adolescents' perceptions of their families' functioning. It may be that, as their families are the only models they have, the incest victims see the distorted family interactions in their own families as normal (Sgroi, 1982). Incest families are often "enmeshed" and very secretive. These families attempt to keep the children in the family from making friends outside of the family in order to prevent the victim from telling about the abuse or leaving the family. Thus, victims have no other family to compare with their own, and so believe that their family is like others (Finkelhor, 1979; Sgroi, 1982).

Furthermore, the findings of the present study did not provide support for a relationship between type of abuse experienced and the adolescent's perception of family functioning. There were no differences found between abuse category and scores on any of the 7 FAD dimensions in this study, although physical and sexual abuse have been shown to be related to family functioning in the literature (Hoagwood \& Stewart, 1989; Harter, Alexander, \& Neimeyer, 1988; Kratcoski, 1984). It is possible that as all subjects were hospitalized, the families in all groups were experiencing dysfunction and thus, this confounded the results. This supposition is supported by the fact that very few adolescents received a score of less than 2 on any 
of the FAD scales, indicating that all adolescents perceived at least some dysfunction as being present in their families.

Looking at the FAD by scales interaction, a relationship was found, however, between adolescents' perceptions of their families' functioning and their personality functioning. Using the general functioning scale of the FAD, adolescents were divided into 3 categories of increasing family dysfunction. While not all scales had significant differences, the general trend was for the high family dysfunction group to report the most difficulties on the MMPI, the low family dysfunction group reported the least problems, while the medium dysfunction group scored in between the other two groups.

Subjects who rated their families as being in the high dysfunction group had significant differences on the $L$, $F$, K, Pd, Pa, Pt, Sc, and Ma Scales from the low family dysfunction group. They also scored significantly higher than the medium dysfunction group on the F and Pd Scales, and the medium dysfunction group scored significantly different from the low dysfunction group on the $\mathrm{K}, \mathrm{Pd}, \mathrm{Pa}$, Pt, Sc, and Ma Scales. In general, these results suggest that the more family dysfunction perceived; the more hostility, paranoia, unconventional thinking, hypomania, anxiety, and psychic pain; the adolescent experiences. The low family dysfunction group reported the least problems. Thus, these results provide support for the literature that 
there is a relationship between family functioning and individual personality functioning (Wyatt \& Newcomb, 1990; Fromuth, 1986; Harter, 1986; Miller, et al., 1986; Tsai, et al., 1979).

Despite the finding of this study that adolescents, who experienced both types of abuse or who rated their families as being highly dysfunctional, scored higher on the MMPI than their comparison groups; few subjects actually scored within the clinically defined pathological range (which is greater than 70 ) on most clinical scales. Although all of the present sample was hospitalized (indicating that the all subjects were having significant difficulties functioning in their home environments), few adolescents possessed MMPI profiles with scaled scores above 70. The mean profile for the group which experienced both physical and sexual abuse had only 1 Clinical scale within the pathological range (above 70 ) which was more than any of the other three groups.

However, as the Chi Square analysis indicated, relationships between abuse category and clinically defined pathology on the F, K, Pt, and Sc Scales were found, indicating that whether adolescents scored above 70 on these scales was related to whether they had been abused and what type of abuse they had experienced. In other words, more adolescents in the abuse categories were experiencing difficulties with emotional pain, anxiety; and unconventional thinking which were severe enough to be 
considered a clinical issue than adolescents who had not experienced abuse.

The fact remains, however, that few adolescents score within the clinically defined pathological ranges on the MMPI despite being hospitalized. This finding is consistent with the literature, as other investigators have found that adolescents do not score as extreme on the MMPI as adults (Goldwater \& Duffy, 1990; Archer, 1987; Scott \& Stone, 1986). For example, scott and Stone's, 1986 study compared the MMPI profiles of a group of 27 adolescent female incest victims, to 31 adult female incest victims. All molested victims were abused by a father or fatherfigure, and were in outpatient therapy at the time of testing. The results of scott and Stone's investigation indicated that the adult victims' mean profile was more elevated than the adolescents' mean profile. They found that the adults possessed two clinical scales, Pd and Sc, above 70, while only the Sc scale fell above 70 for the adolescents.

Several theories have been developed in order to explain this phenomenon. First, adolescents tend to be hospitalized, not because they ask to be or because they are experiencing difficulties, but because they are causing problems for others (Nieminen \& Matson, 1989). Therefore, they may not recognize that they are having problems so they do not report them. In addition, hospitalized adolescents may attempt to suppress unfavorable information 
about themselves in order to shorten their hospital stay, whereas adults are more likely to be admitted voluntarily and thus, more likely to admit to and recognize their difficulties, resulting in higher scores on the MMPI.

Furthermore, in Goldwater and Duffy's (1990) study with adult women, the abuse (sexual or physical, or an alcoholic caretaker) group scored 30 points lower on the Mf scale than on the Pd and Pa scale, and scored significantly higher than the comparison groups on the F, D, and Sc Scales. The adolescents in the present study did not have scores as extreme, although they did present a similar profile. Scott and Stone (1986a) suggested that adolescents do not exhibit the same elevation on the $D$ scale as adults because the abuse arrests development and related identity confusion at the "core" of the personality. They suggest that the elevated $D$ comes from living with this damage for years and is why it may be found with adults but not adolescents.

Finding that abused adolescents have an elevated Pd scale while adults often have an elevated D (Goldwater \& Duffy, 1990) score is not unusual as Pd, psychopathic deviance, has often been described as depression, directed outwards. Depressed adolescents often express their feelings of depression outwardly as aggression, anger, and irritability. Adolescents often do not have the verbal skills or insight necessary to describe the feelings of depression that they are experiencing and thus act out the 
feelings, e.g., irritability, low self-worth, etc. This phenomenon has also been called "masked depression" by researchers and clinicians (Marriage, Fine, Moretti, \& Haley, 1986; Carlson \& Cantwell, 1980; Glaser, 1966).

In addition, Scott and Flowers (1988) generalized Scott and Stone's findings to include not only the $D$ Scale but all other Scales on the MMPI as well. They concluded that the damage from abuse to the personality occurs over time, as the recently abused adolescent incest victims in their study did not demonstrate as much pathology on the MMPI as their adult victims who were molested as children.

Due to the findings that adolescents do not appear to score as extremely as adults on the MMPI, Archer (1987) suggested that 65 might be a more appropriate and useful cut-off for adolescents than 70 . Using 65 as the cut-off to define clinical pathology, the mean profile for adolescents in the present study, who experienced both physical and sexual abuse, had 4 Clinical Scales which fell within the pathological range. In addition, the sexual abuse group had 3 Clinical Scales which fell above the pathological range.

The relationship between abuse category and pathology was again investigated using 65 to define pathology. This time a relationship was found between abuse category and whether one was considered pathological on the F, K, Ma, and Sc Scales. In other words, more adolescents in the abuse categories were experiencing difficulties with 
emotional pain, mania and unconventional thinking which were severe enough to be considered a clinical issue than adolescents who had not experienced abuse.

The findings of these Chi Square analyses suggest that lowering the cut-off point to 65 , to define pathology, may provide a more accurate description of the adolescents' difficulties, as one would expect clinically elevated profiles in a clinical sample. Moreover, when using a cutoff score of 70, a relationship between abuse category and pathology on the Pt Scale was found; at 65 this relationship was not found, although one between pathology on the Pa Scale and abuse category was discovered. Since many previous studies found a relationship between an elevated $\mathrm{Pa}$ Scale and abuse in their studies (especially with adolescents), lowering the cut-off point to 65 may allow "real" relationships to be found, which are hidden when 70 is used, making it easier for clinicians to determine the correlates and causes of adolescents' difficulties (Scott \& Stone, 1986; Scott \& Stone, 1986a). Therefore, these findings provide support for Archer's (1987) suggestion that a cut-off score of 65 for adolescents is more useful than 70 . Moreover, agreement with Archer's conclusion that the MMPI profiles of adolescents should be interpreted with flexibility was indicated, as Archer (1987) stated that adolescence is a time of great emotional turmoil for all adolescents even "normal" ones. 
In addition, MMPI profiles have also been described using code types. A code type is a description of a profile, using the two highest clinical scales, falling above the clinical cut-off score. In this study, the mean profiles for the both and the sexual abuse only groups produced $4 / 8$ (Pd/Sc) profiles, using 65 as the cut-off score. This $4 / 8$ code type is similar to the code types found in other studies (Goldwater \& Duffy, 1990; Scott \& Flowers, 1988; Scott \& Stone, 1986) which have also possessed $4 / 8$ profiles as the mean or most common profile for their abused groups. Individuals with $4 / 8$ code types have typically been described as suffering from identity confusion, and feelings of alienation and vulnerability, which are likely to be acted out in antisocial and hostile behaviors. They also tend to be angry, immature, impulsive, and have marginal social adjustment. These individuals have also been described as "difficult" (Archer, 1987; Duckworth \& Anderson, 1986). It is important to note that $\mathrm{SC}$ is a measure of unconventional or disorganized thinking, not a measure of schizophrenia. Investigators have also found that abused adolescents sometimes have an elevated $8 / 9$ profile (Scott \& Flowers, 1988; Scott \& Stone, 1986; Scott \& Stone, 1986a). Individuals with an $8 / 9$ profile have been described as being hostile, impulsive, argumentative, and paranoid. Moreover, they often act-out in unpredictable ways. They tend to have a labile mood, unrealistic hostility, and 
experience considerable confusion (Duckworth \& Anderson, 1986). Archer (1987) related the occurrence of the $8 / 9$ code type with serious psychopathology. Thus, adolescents with an $8 / 9$ profile have similar presentations to adolescents with $4 / 8$ profiles and may be expressing the same underlying feelings in a slightly different manner. However, only about $6 \%$ of the sample in the present study possessed profiles with either $4 / 8$ or $8 / 9$ code types. of these approximately 30 individuals, there did not appear to be a relationship between abuse category and profile type. However, the present study did find that adolescents who were physically and sexually abused scored significantly higher on the Pd, Sc, and Ma Scales than the other three groups, suggesting that although these may not be the most elevated scales, there does appear to be a relationship between abuse and distress on these three scales. Therefore, the descriptions of the individuals who possess $4 / 8$ and $8 / 9$ code types may also be useful in depicting the abused adolescent. In addition, these findings suggest that an individual's profile should be interpreted using more than just the two highest scales, providing further support for Archer (1987) that adolescents' profiles should be interpreted with flexibility.

The findings also support Heims and Kaufman (1963) who found that the mean MMPI profile for both incest and nonincest victims was $4 / 8$, and thus, they believed that it 
should not be labeled as an incest profile. Heims and Kaufman did conclude that their incest victims endorsed MMPI items, indicating that they were experiencing sexual problems. Meiselman (1980) also found that the profiles of adult female incest and nonincest victims were very similar, although control subjects were less elevated on the Pd and Sc Scales than the incest victims. Meiselman also concluded that her subjects endorsed more items indicating the presence of sexual problems.

Since abused individuals appear to respond to questions on the MMPI differently than nonabused subjects (Meiselman, 1980), it was suggested that the MMPI could be used to predict which individuals had experienced histories of abuse (Roland, 1990). Roland (1990) identified 63 MMPI items which correctly classified $84 \%$ of his sample of adult female outpatients as either sexually abused or not abused. The present study closely replicated the results of Roland, finding that 27 of the 63 items, specified by Roland, could correctly classify 75.38 of sexually abused adolescent female subjects from nonsexually abused females. The items which were included in the analyses focused on self-esteem, self-efficacy, social skills, social isolation, feelings of inadequacy, and of being different or damaged, family dysfunction, and sexuality. Not coincidentally, researchers investigating the effects of sexual abuse have found that abuse leaves adolescents feeling socially isolated, physically damaged, with poor social skills, and 
impaired self-esteem (Beck \& van der Kolk, 1987; Sedney \& Brooks, 1984; Sgroi, 1982; Curtois, 1979; Finkelhor, 1979). Males were also included in the present analyses and an attempt was made to categorize the subjects into one of the four abuse categories. In this case, $52.5 \%$ of the adolescents were correctly classified into one of the four abuse groups, using 31 of the items. Since the specific items proved to be good predictors of abuse category for both males and females, it was concluded that the MMPI Scales should also be able to discriminate between the groups .

Finally, the 14 Scales on the MMPI were used in order to attempt to classify the adolescents into one of the four abuse groups. Scores on the F, Hy, Si, and Mf Scales correctly classified $40.3 \%$ of the subjects into one of the four abuse groups. Many of the specific items which proved to be good predictors in the previous two analyses are actually some of the items which compose the above scales, suggesting that abused adolescents endorse MMPI items indicating that they tend to be female and are experiencing unconventional, distorted perceptions, anxiety, sexual and identity problems, and social isolation. The results of all three of these analyses suggest that specific items on the MMPI are the best predictors of which abuse group the individual belongs more so than their scores on the clinical and validity scales. 
These findings are in accordance with Meiselman (1980) who found that sexual abuse was related to specific MMPI items, although Meiselman concluded that the discriminating items focused solely on sexual adjustment. Thus, the results of this analysis are consistent with the literature which has successfully used the MMPI to identify individuals with specific difficulties in functioning (Kashani, et al., 1990).

Furthermore, the present study supports previous research which has found a relationship between abuse and personality functioning in adolescents such as Scott and Stone's, 1986 study which compared the MMPI profiles of a group of 27 adolescent female incest victims to a nonabused control group. All molested victims were abused by a father or father-figure, and were in outpatient therapy at the time of testing. The results of scott and Stone's investigation indicated that the adolescent incest victims produced overall elevated MMPI profiles as compared to the adolescent control group. In other words, the incest victims scored significantly higher on all scales (except Scale $\mathrm{K}$, on which they scored lower; the lower the score the more pathology on this scale) than the control group.

In a follow-up study, scott and Stone (1986a) compared adolescent female incest victims, ranging in age from 15 to 20, who were in outpatient therapy to a nonabused, nonclinical control group and found that the sexually abused group differed significantly from the control group 
on the F, K, Hs, D, Pd, Pa, Pt, Sc, Ma, and Si MMPI Scales. On all dimensions that differences were found, the incest victims reported more difficulties than their nonabused counterparts.

Although, in general, the results of the present study are in accordance with the literature which states that abused individuals experience more difficulties in personality functioning (Goldwater \& Duffy, 1990; Tsai, et al., 1979), and that the presence of both types of abuse typically has more severe consequences for the individual (Shaunesey, et al., 1993; Bryer et al., 1987) than the presence of either type of abuse alone; fewer MMPI Scales showed significant differences between groups in this investigation than in other studies.

There are several reasons which could account for these differences. First, both males and females were included in the present study whereas males were excluded from most of the previous studies, and their responses may have tempered the results. In the present study, females scored significantly higher than males on the $F, D, P d, P t$, and Sc MMPI Scales. These results suggest that the females in the present sample were expressing more unconventional, disorganized thinking than males, that they were also more hostile and depressed, and had lower self-confidence. Males typically score higher on the Mf scale than females, as a high score indicates stereotypical masculine traits, and a low score indicates stereotypical feminine activities 
and traits. The lower score for women may indicate that the females in this sample were endorsing items usually assigned to their sex, as were males. As can be seen from the results above, females scored significantly higher on several of the scales than males, so it is expected that if females alone were used as the sample, more scales would have been elevated.

Secondly, incest victims have typically been the only subjects, with nonfamilial abuse victims and physical abuse victims largely being excluded from or not identified in most of the previous studies. Again, as different abuse categories were included in this study, the differences between the groups on the MMPI scales may have been diminished.

A third factor is that the subjects in the present study were inpatients, whereas most of the previous studies have used outpatients or nonclinical samples (Roland et al., 1989; Scott \& Stone, 1986). It is hypothesized that the adolescent inpatients may have denied or suppressed unfavorable traits on hospital questionnaires in the hopes of shortening their inpatient stay, thereby covering the differences in the clinical scales between the groups.

This study built on the results of previous studies in that it included a larger number of both physically and sexually abused individuals as subjects, as well as including the much forgotten male victims of sexual abuse. 
In addition, it separated incest victims from nonfamilial abuse subjects in order to investigate whether there were differential effects. Most importantly, it focused on adolescent victims in order to investigate and develop new methods for identifying individuals who have experienced sexual and physical abuse, who may be hesitant about coming forward about their abuse.

However, a number of limitations were met during the course of the investigation. Few significant differences were found between the sexual abuse alone victims and nonabused victims on the MMPI despite what other researchers have found. This is possibly due to using an inpatient population, the inclusion of males, and that the sexual abuse victims in previous studies may have also been physical abuse victims. However, the finding that the sexual abuse only group had 3 Clinical Scales above the pathological range and that the $F$ Scale was significantly higher than for the physical and no abuse groups provides support for the finding of Shaunesey, et al., (1993) that the presence of sexual abuse, not just both sexual and physical abuse, is related to later difficulties in personality functioning.

A second limitation was the heterogeneous definition of incest that was used in the present sample. Moreover, many of the subjects had been abused on multiple occasions by different perpetrators, making them ineligible for 
inclusion into the study. Thus, the results are based on a very limited number of individuals in the sample.

A third limitation was the small number of FAD's completed by subjects. Unfortunately, only one of the hospital units consistently had adolescents complete this questionnaire and if more had been completed, more significant differences may have been found using this measure.

It should also be recognized that the generalizability. of the study is limited as well. As only inpatients were included in the sample, it is not possible to extrapolate to those adolescents who have experienced abuse, but at present are not showing maladaptive symptoms. However, as stated previously, this study was designed to investigate the more severely symptomatic adolescents.

Therefore, it is believed that the presence of both physical and sexual abuse may increase difficulties with later personality functioning. The presence of elevations (as compared to nonabused inpatients) on the $\mathrm{F}, \mathrm{Pd}, \mathrm{Sc}$, and Ma MMPI Scales is suggestive of a history of abuse in adolescents. This study also provided additional support that the MMPI is an effective tool for identifying abused individuals on the basis of their responses to a number of items. Moreover, difficulties in personality functioning are also related to more perceived family dysfunction. Although there were no differences in personality functioning for incest and nonfamilial abuse victims, 
incest victims did report fewer problems in family functioning than nonfamilial abuse victims. However, this finding is believed to be due to the adolescents' desire to cover the abuse and the true extent of the family's problems. In addition, hospitalized adolescents often do not score within the pathological range and therefore, the clinician should view profiles with flexibility in order to effectively utilize the information they provide.

It is suggested that future research focus on identifying the differences between the different types of incest victims in order to ascertain what factors differentiate between them. Nash, Hulsey, Sexton, Harralson, and Lambert (1993) recently completed a study which suggested that adult pathology may be more strongly related to a pathologic home environment rather than to a history of abuse. These findings indicate the need for more research which includes not only measures of abuse but other correlating factors as well in order to determine what the causal factor may be. It would also be helpful to compare a clinical inpatient population with an abused nonclinical outpatient population in order to identify the differences between these groups. Furthermore, indicators of severity of abuse, i.e., sexual act, amount of force used, duration, frequency, etc., should be included in future investigations in order to obtain a fuller, more complete picture of the effects of abuse on adolescents (Tsai et al., 1979). 
Archer, R.P. (1987). Using the MMPI with adolescents. Hillsdale, New Jersey. Lawrence Erlbaum Associates, Publishers.

Asarnow, J.R. \& Carlson, G. (1988). Suicide attempts in preadolescent child psychiatry inpatients. Suicide and Life Threatening Behavior, 18, 129-136.

Beck, J.C. \& van der Kolk, B. (1987). Reports of childhood incest and current behavior of chronically hospitalized psychotic women. American Journal of Psychiatry, 144, 1474-1476.

Blumberg, M.I. (1981). Depression in abused and neglected children. American Journal of Psychotherapy, 35, 342355 .

Briere, J. \& Runtz, M. (1986). Suicidal thoughts and behaviors in former sexual abuse victims. Canadian Journal of Behavioral Sciences, 18, 413-423.

Bryer, J.B., Nelson, B.A., Miller, J.B., Krol, P.A. (1987). Childhood sexual and physical abuse as factors in adult psychiatric illness. American Journal of Psychiatry, 144, 1426-1430.

Byles, J., Byrne, C., Boyle, M.H., \& Offord, D.R. (1988). Ontario Child Health Study: Reliability and Validity of the General Functioning Subscale of the McMaster family assessment device. Family Process, 27, 97-104.

Carlson, G.A. \& Cantwell, D.P. (1980). Unmasking masked depression in children and adolescents. American Journal of Psychiatry, 147, 445-449.

Carmen, E., Rieker, P.P., \& Mills, T. (1984). Victims of violence and psychiatric illness. American Journal of Psychiatry, 141, 378-383.

Clopton, J.R., Post, R.D., \& Larde, J. (1983). Identification of suicide attempters by means of MMPI profiles. Journal of Clinical Psychology, 39, 868871 .

Curtois, C.A. (1979). The incest experience and its aftermath. Victimology: An International Journal, $\underline{4}$, $337-347$.

Dahlstrom, W.G., Welsh, G.S., \& Dahlstrom, L.E. (1975). An MMPI Handbook: Volume II: Research Applications, University of Minnesota Press, Minneapolis. 
Duckworth, J.C. \& Anderson, W.P. (1986). MMPI

interpretation manual for counselors and clinicians.

Muncie, Indiana, Accelerated Development Inc.

Epstein, N.B., Baldwin, L.M., \& Bishop, D.S. (1982). The Family Assessment Device. Brown University/Butler Hospital Family Research Program: Butler Hospital, Providence Rhode Island.

Epstein, N.B., Baldwin, L.M., \& Bishop, D.S. (1983). The McMaster Family Assessment Device. Journal of Marital and Family Therapy, 9 , 171-180.

Finkelhor, D. (1979). Sexually Victimized Children. New York, Macmillan Publishing Co., Inc.

Fromuth, M.E. (1986). The relationship of childhood sexual abuse with later psychological and sexual adjustment in a sample of college women. Child Abuse and Neglect, $10,5-15$.

Glaser, K. (1966). Masked depression in children and adolescents. American Journal of Psychotherapy, 21, 565-574.

Goldwater, L. \& Duffy, J.F. (1990). Use of the MMPI to uncover histories of childhood abuse in adult female psychiatric patients. Journal of Clinical Psychology, $\underline{46}, 392-397$.

Green, A.H. (1978). Self-destructive behavior in battered children. American Journal of Psychiatry, 135, 579582 .

Harter, S., Alexander, P., \& Neimeyer, R.A. (1988). Longterm effects of incestuous child abuse in college women: Social adjustment, social cognition, and family characteristics. Journal of Consulting and Clinical Psychology, 56, 5-8.

Heims, L.W. \& Kaufman, I. (1963). Variations on a theme of incest. American Journal of Orthopsychiatry, 33, 311312 .

Herman, J.I., Perry, C., \& van der Kolk, B.A. (1989). Childhood trauma in borderline personality disorder. American Journal of Psychiatry, 146, 490-495.

Hoagwood, K. \& Stewart, J.M. (1989). Sexually abused children's perceptions of family functioning. Child and Adolescent Social Work Journal, 6, 139-149.

Hollingshead, A.B. (1975). Four Factor Index of Social Status. Department of Sociology, Yale University. 
Husain, A. \& Chapel, J.L. (1983). History of Incest in girls admitted to a psychiatric hospital. American Journal of Psychiatry, 140, 591-593.

Kabacoff, R.I., Miller, I.W., Bishop, D.S., Epstein, N.B., Keitner, G.I. (1990). A psychometric study of the McMaster family assessment device in psychiatric, medical, and nonclinical samples. Journal of Family Psychology, $\underline{3}, 431-439$.

Kadushin, A. \& Martin, J.A. (1981). Child Abuse: An Interactional Event. New York, Columbia University Press.

Kashani, J.H., Dandoy, A.C., Vaidya, A.F., Soltys, S.M., \& Reid, J.C. (1990). Risk factors and correlates of severe psychiatric disorders in a sample of inpatient children. American Journal of Psychiatry, 147, 780784 .

Keitner, G.I., Miller, I.W., Epstein, N.B., \& Bishop, D.S. $(1986)$. The functioning in families of patients with major depression. International Journal of Family Psychiatry, $\underline{7}, 11-16$.

Kempe, C.H. \& Helfer, R.E. The Battered Child. (1980). Chicago, The University of Chicago Press.

Kratcoski, P.C. (1984). Perspectives on intrafamily violence. Human Relations, 37, 443-453.

Marks, P.A., Seeman, W., \& Haller, D.L. (1974). The actuarial use of the MMPI with adolescents and adults. Baltimore: Williams \& Wilkins.

Marriage, K., Fine, S., Moretti, M., \& Haley, G. (1986). Relationship between depression and conduct disorder in children and adolescents. Journal of the American Academy of Child Psychiatry, 25, 687-691.

Mayhall, P.D. \& Norgard, K.E. (1983). Child Abuse and Neglect. New York, John Wiley \& Sons.

Meiselman, K.C. (1980). Personality characteristics of incest history in psychotherapy patients: A research note. Archives of Sexual Behavior, 9, 195-197.

Miller, I.W., Kabacoff, R.I., Keitner, G.I., Epstein, N.B., \& Bishop, D.S. (1986). Family functioning in the families of psychiatric patients. Comprehensive Psychiatry, 27, 302-312. 
Nash, M.R., Hulsey, T.L., Sexton, M.C., Harralson, T.L., \& Lambert, W. (1993). Long-term sequelae of childhood sexual abuse: Perceived family environment, psychopathology, and dissociation. Journal of Consulting and Clinical Psychology, 61, 276-283.

Neiger, B.L. \& Hopkins, R.W. (1988). Adolescent Suicide: Character traits of high-risk teenagers. Adolescence, $\underline{23}, 469-475$.

Nieminen, G.S. \& Matson, J.L. (1989). Depressive problems in conduct-disordered adolescents. Journal of School Psychology, 27, 175-188.

Plummer, B.A., Gispert, M., Hayden, R.M. Robbins, D., \& Seifer, R. (1989). Depression, Hopelessness, and Substance Abuse in Hospitalized Adolescents with Suicidal Ideation or Behavior. Paper presented at the 15th Congress of the International Association for Suicide Prevention, Brussels, Belgium.

Roland, B.C., Zelhart, P.F., \& Dubes, R. (1988). Selected MMPI items that identified college women who reported early sexual abuse. Psychological Reports, 63, 447450 .

Roland, B.C., Zelhart, P.F., \& Dubes, R. (1989). MMPI correlates of college women who reported experiencing child/adult sexual contact with father, stepfather, or with other persons. Psychological Reports, 64, 11591162 .

Russell, D.E.H. (1986). The Secret Trauma: Incest in the Lives of Girls and Women. New York, Basic Books, Inc., Publishers.

Sansonnet-Hayden, H., Haley, G., Marriage, K., \& Fine, S. (1986). Sexual abuse and psychopathology in hospitalized adolescents. Journal of the American Academy of Child and Adolescent Psychiatry, 26, 753757 .

Scott, R.L. \& Flowers, J.V. (1988). Betrayal by the mother as a factor contributing to psychological disturbance in victims of father-daughter incest: An MMPI analysis. Journal of Social and Clinical Psychology, $\underline{6}, 147-154$.

Scott, R.L. \& Stone, D.A. (1986). MMPI profile constellations in incest families. Journal of Consulting and Clinical Psychology, 54, 364-368. 
Scott, R.I. \& Stone, D.A. (1986a). MMPI measures of psychological disturbance in adolescent and adult victims of father-daughter incest. Journal of Clinical Psychology, 42, 251-259.

Sedney, M.A. \& Brooks, B. (1984). Factors associated with a history of childhood sexual experience in a nonclinical female population. Journal of the American Academy of Child Psychiatry, 23, 215-218.

Sgroi, S. (1982). Handbook of Clinical Intervention in Child Sexual Abuse. Lexington, MA: Lexington Books.

Shaunesey, K., Cohen, J.L., Plummer, B., \& Berman, A. (1993). Suicidality in hospitalized adolescents: Relationship to prior abuse. Journal of Orthopsychiatry, 63, 113-119.

Spirito, A., Faust, D., Myers, B., \& Bechtel, D. (1988). Clinical utility of the MMPI in the evaluation of adolescent suicide attempters. Journal of Personality Assessment, 52, 204-211.

Spirito, A., Overholser, J., \& Stark, L.J. (1988). Common Problems and coping strategies II: Findings with adolescent suicide attempters. Journal of Abnormal Child Psychology, 17, 213-221.

Spirito, A., Stark, L., Fristad, M., Hart, K., \& OwensStively, J. (1987). Adolescent suicide attempters hospitalized on a pediatric unit. Journal of Pediatric Psychology, 12, 171-189.

Starr, R.H. (1988). Physical Abuse of Children in Handbook of Family Violence. New York, Plenum Press.

Tarter, R.E., Hegedus, A.M., Winsten, N.E., \& Alterman, A.I. (1984). Neuropsychological, Personality, and Familial Characteristics of physically abused delinquents. Journal of the American Academy of Child Psychiatry, 23, 668-674.

Tsai, M., Feldman-Summers, S., \& Edgar, M. (1979). Childhood molestation: Variables related to differential impacts on psychosexual functioning in adult women. Journal of Abnormal Psychology, 88, 407417 .

Williams. C.L. \& Butcher, J.N. (1989). An MMPI study of adolescents: I. Empirical validity of the standard scales. Psychological Assessment, 1, 251-259. 
Wyatt, G.E. (1985). The sexual abuse of Afro-American and white-American women in childhood. Child Abuse and Neglect, $\underline{9}$, 507-519.

Wyatt, G.E. \& Peters, S.D. (1986). Issues in the definition of child sexual abuse in prevalence research. Child Abuse and Neglect, 10, 231-240.

Wyatt, G.E. \& Newcomb, M. (1990). Internal and external mediators of women's sexual abuse in childhood. Journal of Consulting and Clinical Psychology, 58, 758-767. 
Archer, R.P. (1987). Using the MMPI with adolescents. Hillsdale, New Jersey. Lawrence Erlbaum Associates, Publishers.

Asarnow, J.R. \& Carlson, G. (1988). Suicide attempts in preadolescent child psychiatry inpatients. Suicide and Life Threatening Behavior, 18, 129-136.

Beck, J.C. \& van der Kolk, B. (1987). Reports of childhood incest and current behavior of chronically hospitalized psychotic women. American Journal of Psychiatry, 144, 1474-1476.

Blumberg, M.L. (1981). Depression in abused and neglected children. American Journal of Psychotherapy, 35, 342355 .

Briere, J. \& Runtz, M. (1986). Suicidal thoughts and behaviors in former sexual abuse victims. Canadian Journal of Behavioral Sciences, 18, 413-423.

Bryer, J.B., Nelson, B.A., Miller, J.B., Krol, P.A. (1987). Childhood sexual and physical abuse as factors in adult psychiatric illness. American Journal of Psychiatry, 144, 1426-1430.

Byles, J., Byrne, C., Boyle, M.H., \& Offord, D.R. (1988). Ontario Child Health Study: Reliability and Validity of the General Functioning Subscale of the McMaster family assessment device. Family Process, 27, 97-104.

Carlson, G.A. \& Cantwell, D.P. (1980). Unmasking masked depression in children and adolescents. American Journal of Psychiatry, 147, 445-449.

Carmen, E., Rieker, P.P., \& Mills, T. (1984). Victims of violence and psychiatric illness. American Journal of Psychiatry, 141, 378-383.

Clopton, J.R., Post, R.D., \& Larde, J. (1983). Identification of suicide attempters by means of MMPI profiles. Journal of Clinical Psychology, 39, 868871 .

Curtois, C.A. (1979). The incest experience and its aftermath. Victimology: An International Journal, $\underline{4}$, 337-347.

Dahlstrom, W.G., Welsh, G.S., \& Dahlstrom, L.E. (1975). An MMPI Handbook: Volume II: Research Applications, University of Minnesota Press, Minneapolis. 
Duckworth, J.C. \& Anderson, W.P. (1986). MMPI

interpretation manual for counselors and clinicians.

Muncie, Indiana, Accelerated Development Inc.

Epstein, N.B., Baldwin, L.M., \& Bishop, D.S. (1982). The Family Assessment Device. Brown University/Butler Hospital Family Research Program: Butler Hospital, Providence Rhode Island.

Epstein, N.B., Baldwin, L.M., \& Bishop, D.S. (1983). The McMaster Family Assessment Device. Journal of Marital and Family Therapy, $\underline{9}, 171-180$.

Finkelhor, D. (1979). Sexually Victimized Children. New York, Macmillan Publishing Co., Inc.

Fromuth, M.E. (1986). The relationship of childhood sexual abuse with later psychological and sexual adjustment in a sample of college women. Child Abuse and Neglect, $10,5-15$.

Glaser, K. (1966). Masked depression in children and adolescents. American Journal of Psychotherapy, 21, 565-574.

Goldwater, L. \& Duffy, J.F. (1990). Use of the MMPI to uncover histories of childhood abuse in adult female psychiatric patients. Journal of Clinical Psychology, $\underline{46}, 392-397$.

Green, A.H. (1978). Self-destructive behavior in battered children. American Journal of Psychiatry, 135, 579582 .

Harter, S., Alexander, P., \& Neimeyer, R.A. (1988). Longterm effects of incestuous child abuse in college women: Social adjustment, social cognition, and family characteristics. Journal of Consulting and Clinical Psychology, 56, 5-8.

Heims, L.W. \& Kaufman, I. (1963). Variations on a theme of incest. American Journal of Orthopsychiatry, 33, 311312 .

Herman, J.L., Perry, C., \& van der Kolk, B.A. (1989). Childhood trauma in borderline personality disorder. American Journal of Psychiatry, 146, 490-495.

Hoagwood, K. \& Stewart, J.M. (1989). Sexually abused children's perceptions of family functioning. Child and Adolescent Social Work Journal, 6 , 139-149.

Hollingshead, A.B. (1975). Four Factor Index of Social Status. Department of Sociology, Yale University. 
Husain, A. \& Chapel, J.L. (1983). History of Incest in girls admitted to a psychiatric hospital. American Journal of Psychiatry, 140, 591-593.

Kabacoff, R.I., Miller, I.W., Bishop, D.S., Epstein, N.B., Keitner, G.I. (1990). A psychometric study of the McMaster family assessment device in psychiatric, medical, and nonclinical samples. Journal of Family Psychology, 3, 431-439.

Kadushin, A. \& Martin, J.A. (1981). Child Abuse: An Interactional Event. New York, Columbia University Press.

Kashani, J.H., Dandoy, A.C., Vaidya, A.F., Soltys, S.M., \& Reid, J.C. (1990). Risk factors and correlates of severe psychiatric disorders in a sample of inpatient children. American Journal of Psychiatry, 147, 780784 .

Keitner, G.I., Miller, I.W., Epstein, N.B., \& Bishop, D.S. (1986). The functioning in families of patients with major depression. International Journal of Family Psychiatry, $\underline{7}, 11-16$.

Kempe, C.H. \& Helfer, R.E. The Battered Child. (1980). Chicago, The University of Chicago Press.

Kratcoski, P.C. (1984). Perspectives on intrafamily violence. Human Relations, 37, 443-453.

Marks, P.A., Seeman, W., \& Haller, D.L. (1974). The actuarial use of the MMPI with adolescents and adults. Baltimore: Williams \& Wilkins.

Marriage, K., Fine, S., Moretti, M., \& Haley, G. (1986). Relationship between depression and conduct disorder in children and adolescents. Journal of the American Academy of Child Psychiatry, 25, 687-691.

Mayhall, P.D. \& Norgard, K.E. (1983). Child Abuse and Neglect. New York, John Wiley \& Sons.

Meiselman, K.C. (1980). Personality characteristics of incest history in psychotherapy patients: A research note. Archives of Sexual Behavior, 9 , 195-197.

Miller, I.W., Kabacoff, R.I., Keitner, G.I., Epstein, N.B., \& Bishop, D.S. (1986). Family functioning in the families of psychiatric patients. Comprehensive Psychiatry, 27, 302-312. 
Nash, M.R., Hulsey, T.L., Sexton, M.C., Harralson, T.L., \& Lambert, W. (1993). Long-term sequelae of childhood sexual abuse: Perceived family environment, psychopathology, and dissociation. Journal of Consulting and Clinical Psychology, 61, 276-283.

Neiger, B.L. \& Hopkins, R.W. (1988). Adolescent Suicide: Character traits of high-risk teenagers. Adolescence, $23,469-475$.

Nieminen, G.S. \& Matson, J.L. (1989). Depressive problems in conduct-disordered adolescents. Journal of School Psychology, 27, 175-188.

Plummer, B.A., Gispert, M., Hayden, R.M. Robbins, D., \& Seifer, R. (1989). Depression, Hopelessness, and Substance Abuse in Hospitalized Adolescents with Suicidal Ideation or Behavior. Paper presented at the 15th Congress of the International Association for Suicide Prevention, Brussels, Belgium.

Roland, B.C., Zelhart, P.F., \& Dubes, R. (1988). Selected MMPI items that identified college women who reported early sexual abuse. Psychological Reports, 63, 447450 .

Roland, B.C., Zelhart, P.F., \& Dubes, R. (1989). MMPI correlates of college women who reported experiencing child/adult sexual contact with father, stepfather, or with other persons. Psychological Reports, 64, 11591162 .

Russell, D.E.H. (1986). The Secret Trauma: Incest in the Lives of Girls and Women. New York, Basic Books, Inc., Publishers.

Sansonnet-Hayden, H., Haley, G., Marriage, K., \& Fine, S. (1986). Sexual abuse and psychopathology in hospitalized adolescents. Journal of the American Academy of Child and Adolescent Psychiatry, 26, 753757.

Scott, R.L. \& Flowers, J.V. (1988). Betrayal by the mother as a factor contributing to psychological disturbance in victims of father-daughter incest: An MMPI analysis. Journal of Social and Clinical Psychology, 6, $147-154$.

Scott, R.L. \& Stone, D.A. (1986). MMPI profile constellations in incest families. Journal of Consulting and Clinical Psychology, 54, 364-368. 
Scott, R.L. \& Stone, D.A. (1986a). MMPI measures of psychological disturbance in adolescent and adult victims of father-daughter incest. Journal of Clinical Psychology, 42, 251-259.

Sedney, M.A. \& Brooks, B. (1984). Factors associated with a history of childhood sexual experience in a nonclinical female population. Journal of the American Academy of Child Psychiatry, 23, 215-218.

Sgroi, S. (1982). Handbook of Clinical Intervention in Child Sexual Abuse. Lexington, MA: Lexington Books.

Shaunesey, K., Cohen, J.L., Plummer, B., \& Berman, A. (1993). Suicidality in hospitalized adolescents: Relationship to prior abuse. Journal of Orthopsychiatry, 63, 113-119.

Spirito, A., Faust, D., Myers, B., \& Bechtel, D. (1988). Clinical utility of the MMPI in the evaluation of adolescent suicide attempters. Journal of Personality Assessment, 52, 204-211.

Spirito, A., Overholser, J., \& Stark, L.J. (1988). Common Problems and coping strategies II: Findings with adolescent suicide attempters. Journal of Abnormal Child Psychology, 17, 213-221.

Spirito, A., Stark, L., Fristad, M., Hart, K., \& OwensStively, J. (1987). Adolescent suicide attempters hospitalized on a pediatric unit. Journal of Pediatric Psychology, 12, 171-189.

Starr, R.H. (1988). Physical Abuse of Children in Handbook of Family Violence. New York, Plenum Press.

Tarter, R.E., Hegedus, A.M., Winsten, N.E., \& Alterman, A.I. (1984). Neuropsychological, Personality, and Familial Characteristics of physically abused delinquents. Journal of the American Academy of Child Psychiatry, 23, 668-674.

Tsai, M., Feldman-Summers, S., \& Edgar, M. (1979) . Childhood molestation: Variables related to differential impacts on psychosexual functioning in adult women. Journal of Abnormal Psychology, 88, 407417 .

Williams. C.L. \& Butcher, J.N. (1989). An MMPI study of adolescents: I. Empirical validity of the standard scales. Psychological Assessment, 1, 251-259. 
Wyatt, G.E. (1985). The sexual abuse of Afro-American and white-American women in childhood. Child Abuse and Neglect, $\underline{9}, 507-519$.

Wyatt, G.E. \& Peters, S.D. (1986). Issues in the definition of child sexual abuse in prevalence research. Child Abuse and Neglect, $10,231-240$.

Wyatt, G.E. \& Newcomb, M. (1990). Internal and external mediators of women's sexual abuse in childhood. Journal of Consulting and Clinical Psychology, 58, 758-767. 